\title{
Study of the stability of a large realistic cyclonic eddy
}

de Marez Charly ${ }^{1,}{ }^{*}$, Meunier Thomas ${ }^{2}$, Morvan Mathieu ${ }^{1}$, L'hégaret Pierre ${ }^{1}$, Carton Xavier ${ }^{1}$

\author{
1 Univ. Brest, Laboratoire d'Océanographie Physique et Spatiale (LOPS), IUEM, Rue Dumont D'urville, \\ 29280 Plouzané, France \\ ${ }^{2}$ CICESE, Ensenada, B.C., Mexico \\ *Corresponding author : Charly de Marez, email address : charly.demarez@univ-brest.fr
}

\begin{abstract}
:
We investigate the stability of a composite cyclone representative of Arabian Sea eddies using a high resolution primitive equation model. We observe that the eddy is unstable with respect to a mixed barotropic/baroclinic instability, leading to the growth of an azimuthal mode 2 perturbation. The latter deforms the eddy, which eventually evolves into a tripole after about 4 months of simulation. The presence of a critical level for the most unstable mode generates sharp fronts in the surface mixed layer where the Rossby number is large. These fronts then become unstable, and this generates submesoscale cyclones and filaments. Near these fronts, diapycnal mixing occurs, causing the potential vorticity to change sign locally, and symmetric instability to develop in the core of the cyclonic eddy. Despite the instabilities, the eddy is not destroyed and remains a large-scale coherent structure for the last 6 months of the simulation. Looking at Sea Surface Height only, the composite eddy evolves little, and fairly represents the eddy observed in the altimetry which can live for several months. The study of this simulation thus illustrates the numerous kinds of instabilities which may occur in large cyclonic eddies but can not be captured directly by altimetric data.
\end{abstract}

\section{Highlights}

A composite cyclone representative of the Arabian Sea eddies is unstable with respect to a mixed barotropic/baroclinic instability. Submesoscale features are generated at the surface via barotropic, baroclinic, and symmetric instabilities. Currently available altimetric and floats data cannot capture the instability occurring in the eddy.

Keywords : Mesoscale, Eddy, Instability, Submesoscale, Vortex 


\section{Introduction}

Mesoscale eddies are a prominent feature of the ocean circulation. They have a strong influence on biological activity (Chelton et al., 2011), tracer transport (Zhang et al., 2014), and physical and chemical properties of the water column (Dong et al., 2014). Examples of long lived, coherent, and recurrent mesoscale eddies can be found in every oceanic basin: Gulf Stream rings in the western North Atlantic (Richardson, 1983), meddies in the eastern North Atlantic (Armi et al., 1989), Agulhas rings in the South Atlantic (Olson \& Evans, 1986), Mozambique channel eddies in the Indian Ocean (Halo et al., 2014), Kuroshio rings in the western North Pacific (Li et al., 1998), Loop Current Eddies in the Gulf of Mexico (Meunier et al., 2018), the Ras al Hadd dipole in the northern Arabian Sea (L'Hégaret et al., 2015, 2016). All play an important role in the transport of heat and salt.

Mesoscale eddies have been intensively studied over the past 60 years. In particular, many studies have focused on their stability properties, using linear stability analysis (Gent \& McWilliams, 1986; McWilliams et al., 1986; Nguyen et al., 2012; Yim \& Billant, 2015), and numerical models to study the finite amplitude evolution of unstable perturbations (Carton \& McWilliams, 1989; Stegner \& Dritschel, 2000; Ménesguen et al., 2018). In these studies, eddies are modelled using analytical profiles, e.g shielded vortices, Rankine vortices (Carton \& McWilliams, 1989; Stegner \& Dritschel, 2000)), Gaussian vortices (McWilliams, 1985), or Lorentzian vortices (Hua et al., 2013; Meunier et al., 2018) assuming that they represent a large range of observed eddies (Le Vu et al., 2018). The result of these stability analyses is strongly dependent on the initial profile imposed. In most cases, the eddy profiles are unstable, and can lead to the destruction of the eddy, the formation of dipoles, tripoles, or the long-time decay of the eddy (Carton et al., 1989; Stegner \& Dritschel, 2000).

The increase of computational capabilities has led recent studies to implement very high resolution stability analyses in a 3D context. This allows the study of vortex stability beyond balanced mesoscale processes, and to observe how submesoscale features can emerge from the destabilization of mesoscale structures (Brannigan et al., 2017; Ménesguen et al., 2018). Such studies highlight a possible mechanism for the forward cascade of energy toward small scales.

In the past few years, it has become possible to determine the actual shape of oceanic 
eddies with a fairly good spatio-temporal resolution. Gridded multi-satellite altimetric products provide increased horizontal resolution (up to $1 / 8^{\circ}$ ) and resolve more accurately eddies with radii two or three times the first baroclinic Rossby radius of deformation $\left(R_{D}\right.$ hereafter $)$ than older products with coarser horizontal resolution $\left(1 / 3^{\circ}\right.$ or $\left.1 / 4^{\circ}\right)$. The rapid growth of the Argo database as well as the increase of the resolution of the vertical profiles significantly improved the sampling of the vertical structure of the global ocean. Combining the Argo and altimetry datasets, Chaigneau et al. (2011) have shown that composite methods are efficient in determining the average 3D shape of eddies and isolating classes of eddies in given areas. This effort has been made in several regions, e.g Chaigneau et al. (2011) in the eastern South Pacific Ocean, Keppler et al. (2018) in the Southwest Tropical Pacific ocean, and de Marez et al. (2019) in the Arabian Sea. In the latter study, the authors computed a composite eddy without a priori assumptions (e.g a Gaussian pressure/velocity profile) and only from in situ measurements. Because the composite is a mean of a very varied population, it may not in fact be representative of all individual element of the population. However, through comparison with altimetric and in situ — drifters, floats, and ship - measurements, authors showed that the composite cyclonic eddy is fairly representative of the Arabian Sea's mesoscale cyclones already observed.

In this paper, we investigate the stability of a composite eddy, by running a high resolution primitive equation model initialized with a composite cyclone computed in the Arabian Sea. Contrary to previous studies, we do not use analytical initial conditions. The Arabian Sea, in the northern Indian Ocean, between the African and Asian landmasses, is a region dominated by the monsoon wind regime. This regime leads to seasonal along-shore current reversals. The alongshore currents destabilize and form meanders with 100-200 km wavelengths which eventually detach to form eddies with similar diameters (Schott \& McCreary, 2001). Wind forcing and baroclinic Rossby waves generated at the eastern boundary of the Arabian Sea can strengthen the eddies and contribute to their propagation (Al Saafani et al., 2007). Eddies have a strong influence on the regional circulation. Mesoscale activity is reflected by high values of Eddy Kinetic Energy (Scharffenberg \& Stammer, 2010) and Eddy Available Potential Energy (Roullet et al., 2014), nearly reaching the values in the Antarctic Circumpolar Current or the Agulhas Current. We refer the reader to Fig. 1 in L'Hégaret 
et al. (2018) which presents the mean surface circulation in this area, and Fig. 2 in Vic et al. (2014) for a description of the mean standing eddies there, such as the Great Whirl and the dipole of Ra's Al Hadd.

This is the first time to our knowledge that the non-linear stability study of an isolated eddy is performed using in situ and satellite observed initial conditions. Here, we focus on physical processes underlying the long time evolution of the composite eddy. We address the following questions: (1) Is the composite cyclone stable in the primitive equation framework ? (2) What are the physical mechanisms involved in the destabilization of the eddy ? (3) Can the destabilization process, and the shape of the eddy after destabilization, be observed using altimetry and Argo data.

The paper is organized as follows. We present the composite eddy, and describe the numerical setup and tools used to study the simulation in section 2 . We present the results of the simulations, and describe the diagnostics performed to analyse the possible sources of instability in section 3. We discuss sensitivity tests of our simulations in section 4. Finally, the results are discussed in section 5 .

\section{Context and methods}

\subsection{The composite eddy and its environment}

The composite eddy was extracted using the following procedure: (1) Mesoscale eddies are detected from a $1 / 8^{\circ}$ altimetric product using the AMEDA algorithm (Le Vu et al., 2018) for the period 2000-2015. (2) A colocalization algorithm is then used to detect if an Argo float is located in or out of an eddy. (3) The background stratification of the region (Fig. 1 ) is computed by averaging Argo profiles located outside the eddies (7885 profiles). (4) 531 Argo profiles, collected inside surface-intensified Cyclonic Eddies of radius $R_{D}<R<3 R_{D}$, are isolated. $R_{D}=47 \mathrm{~km}$ is the average First Baroclinic Rossby Radius of Deformation in the area (Chelton et al., 1998). (5) Density profiles are computed from Argo profiles and density anomalies are obtained by subtracting the background density in the area. (6) A dimensionless radial position $\tilde{R}=d R / R$ is computed for each profile, where $d R$ is the distance between the center of the eddy and the position of the colocalized profile $(d R)$, and $R$ is the radius of the eddy. (7) The profiles are averaged depending on their value of $\tilde{R}$, in 

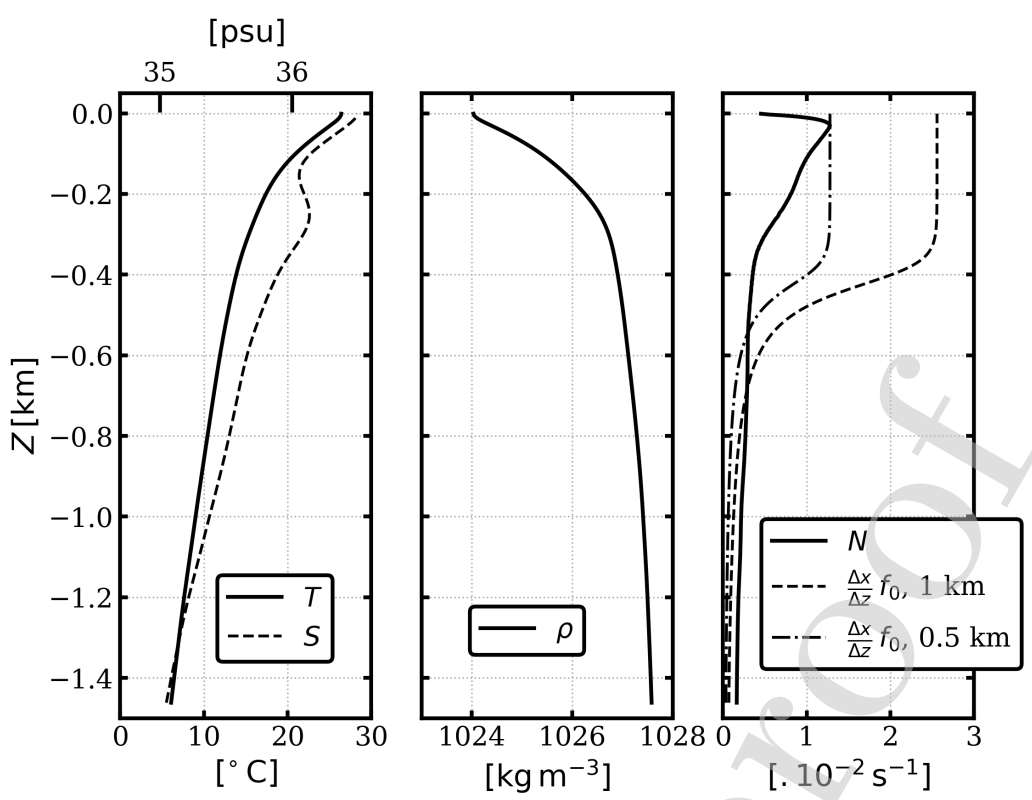

Figure 1: Background temperature (left, solid), salinity (left, dashed), density (middle) and Brunt-Väisälä frequency $N$ (right solid) profiles, after a short period of integration. This corresponds to the stratification as found by Argo floats in the northern Arabian Sea, and used to initialize the simulations, with an additional thin surface mixed layer generated by the KPP scheme during the first days of the simulation (once created its depth is constant throughout time). The right panel shows in dashed lines the ratio $\frac{\Delta x}{\Delta z} f_{0}$ for two simulations, which can be compared to $N ; \Delta x$ and $\Delta z$ are the model grid spacings.

bins of width $\Delta R=0.05$, yielding a composite vertical section of density anomaly $\tilde{\rho}(\tilde{R}, z)$.

For each depth, the density anomaly $\tilde{\rho}(\tilde{R}, z)$ is smoothed using a Gaussian Kernel of width 0.3. (9) Finally, this composite is extrapolated into a 3D structure, by assuming azimuthal symmetry. The horizontal extent of the composite is re-dimensionalized by the mean radius of all eddies used for the computation of the composite $\langle R\rangle=100 \mathrm{~km}$.

The Structure of the composite Cyclonic Eddy is thus defined in terms of a density anomaly distribution. The sum of the density anomaly of the composite and the background stratification is shown in Fig. 2. Following the same procedure, a composite structure of salinity anomaly is computed (Fig. 2b). de Marez et al. (2019) show that these structures are representative of the typical mesoscale eddies observed in the Arabian Sea. Details of the composite Cyclonic Eddy calculation and the associated validation are fully described in de Marez et al. (2019).

We compute the temperature anomaly field from the composite salinity and density, 

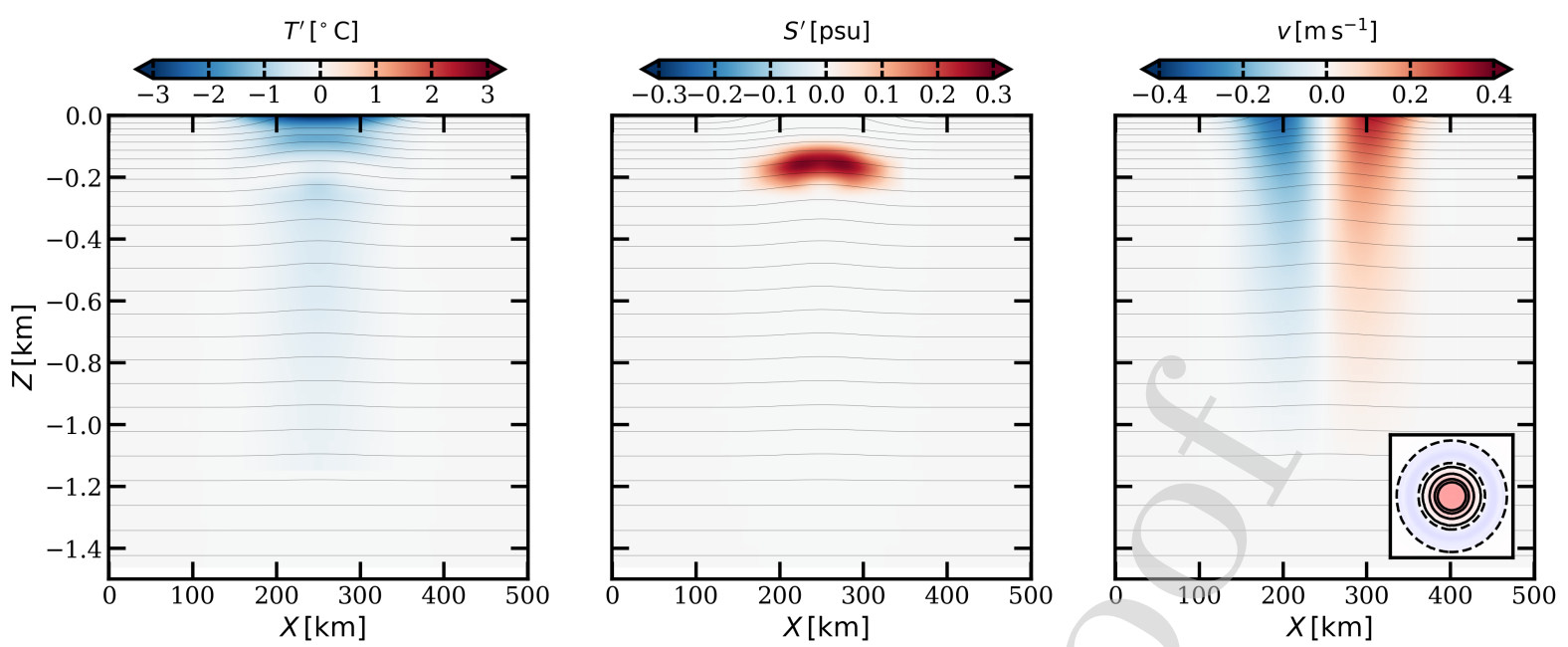

Figure 2: Vertical section at the center of the domain $(y=250 \mathrm{~km})$ at initialization, showing the shape of the composite eddy. (left) Temperature anomaly, (middle) salinity anomaly and (right) meridional (southnorth) velocity $v$. Iso-density contours of the composite eddy, with a spacing of $0.4 \mathrm{~kg} \mathrm{~m}^{-3}$, are superposed in all panels. Insert in the right panel shows the $-0.02,0.02,0.1,0.2$ and 0.25 dynamic Rossby number $\zeta / f_{0}$ contours at the surface; the colormap in the insert is the same as in Fig. 3.

using the Gibbs SeaWater (GSW) Oceanographic Toolbox (McDougall \& Barker, 2011), which uses the TEOS-10 equation of state for seawater (Fig. 2(a)). The background profiles shown in Fig. 1(a) are then added to the temperature and salinity anomaly fields (note that the surface mixed layer is initially not present). The velocity field is obtained via the cyclogeostrophic balance, following the procedure described in Penven et al. (2014), see Fig. 2(right). The numerical simulations are initialized with the temperature, salinity, and velocity fields corresponding to the composite eddy.

It is worth mentioning that by definition, the composite eddy has a coarse resolution. (1) On the horizontal direction: the averages of Argo-collected density profiles are made in bins of size $\Delta R=0.05$. Once re-dimensionalized, this corresponds to a horizontal resolution of the composite eddy of $5 \mathrm{~km}$. Since we perform high resolution numerical simulations, the composite eddy is interpolated on the fine grid of the simulation using a cubic-interpolation. (2) On the vertical direction: while the vertical resolution of Argo float profiles is irregular and $O(10) \mathrm{m}$, they were interpolated on a regularly spaced vertical grid, with a vertical spacing of $4.4 \mathrm{~m}$. The composite is then linearly interpolated on the finer vertical grid of the simulation. In the simulation we will discuss, small scale variations of density and velocity 
are of $O(<5) \mathrm{km}$ on the horizontal and $O(<10) \mathrm{m}$ on the vertical. Such small scale features thus occur at scales that the composite computation could not capture.

The eddy is intensified at the surface, but has a deep-reaching influence down to about $1000 \mathrm{~m}$ depth. Its horizontal shape corresponds to a shielded vorticity monopole: a positive core of vorticity and a shield of negative vorticity (see insert in Fig. 2). Its radius, $R=100$ $\mathrm{km}$, is large compared to the mean regional $R_{D}(47 \mathrm{~km})$. The Burger number, defined as $B u=\left(\frac{R_{d}}{R}\right)^{2}$ scales as $B u=\left(\frac{47}{100}\right)^{2} \sim 0.25<1$. In the following, mentions to 'submesoscale' refer to features and processes occurring at scales that are small compared to $R_{D}$ (i.e. $B u>1)$. Typically, the fronts and vortices generated around the eddy have a typical scale of $O(<10) \mathrm{km}$. They are submesoscale features. The Coriolis parameter is kept constant in the simulations ( $f$-plane), using the regional average value (latitude of $23^{\circ} \mathrm{N}$ ): $f_{0}=5.210^{-5} \mathrm{~s}^{-1}$. The initial condition's Rossby Number is small, $R o=\frac{U_{\max }}{f_{0} R_{\max }} \sim 0.13$, with $U_{\max }$ the maximal velocity, at $R_{\max }$. Thus $R o / B u$ is finite, and the eddy is frontal (Stegner \& Dritschel, 2000). However, it has been shown that the dynamical Rossby number $\epsilon=\zeta / f_{0}$, with $\zeta$ the relative vorticity is a more pertinent parameter to study geostrophic and ageostrophic dynamics in vortices (Stegner \& Dritschel, 2000). In our case, $\epsilon \sim 0.3$ in the core of the eddy at initialization. Finally, the Froude number is $F r=\frac{\epsilon}{\sqrt{B u}} \sim 0.6$.

\subsection{Numerical setup}

To study the dynamics of the composite Cyclonic Eddy described in section 2.1, we perform spindown idealized simulations, with the surface fluxes set to zero, using the Coastal and Regional Ocean COmmunity model, CROCO (Shchepetkin \& McWilliams, 2005). It solves the hydrostatic primitive equations (PE) for the velocity $\mathbf{u}=(u, v, w)$, temperature $T$, and salinity $S$, using a full equation of state for seawater (Shchepetkin \& McWilliams, 2011). The potential density referenced at the surface $\rho$ is computed offline from $\mathrm{T}$ and S using the TEOS-10 equation of state for seawater. From the potential density, we also compute the Brunt-Väisälä frequency defined by

$$
N=\sqrt{-\frac{g}{\rho_{0}} \frac{\partial \rho}{\partial z}},
$$

with $g=9.81 \mathrm{~m} \mathrm{~s}^{-2}$, and $\rho_{0}$ the mean density in the domain. The simulations performed 
integrate the $\mathrm{PE}$ for about 300 days on the $f$-plane, with a time step $d t=120 \mathrm{~s}$, respecting the Courant Friedrichs Lewy (CFL) criterion. The numerical settings are similar to previous simulations performed in idealized contexts (Ménesguen et al., 2018): horizontal advection terms for tracers and momentum are discretized with fifth-order upwind advection schemes (UP5); the horizontal viscosity and diffusivity are set to zero, since the UP5 scheme damps dispersive errors; the vertical advection is discretized with a fourth-order centered parabolic spline reconstruction (Splines scheme); the vertical closure is done by adding a small vertical diffusion in the interior using a K-profile parameterization (KPP) (Large et al., 1994). Further discussion about these parameterizations can be found in Klein et al. (2008) or Ménesguen et al. (2018).

The KPP-scheme generates a thin surface mixed layer during the first days of the simulation. The latter is not present in the background stratification at initialization because it is computed as an average throughout the whole year. Once created (from $t \sim 20$ days), the surface mixed layer does not evolve, and it is consistent with the surface mixed layer observed in the density profiles of the Arabian Sea (except during the winter). It has a vertical extension of about $30 \mathrm{~m}$ depth, and can be seen in Fig. 1.

The domain size is $500 \times 500 \mathrm{~km}$ on the horizontal. Taking a flat bottom at a depth of $1500 \mathrm{~m}$ is enough because the eddy has a vertical extension of about $1000 \mathrm{~m}$. We set open boundary conditions at the edges of the domain. This allows the radiation of gravity waves generated during the initial adjustment of the eddy. These waves are evacuated out of the domain at $t=1$ day.

The vertical and horizontal resolutions are chosen to accurately resolve the frontal dynamics and the forward energy cascade at the surface. The simulation has 256 vertical levels stretched at the surface such that the vertical resolution is $\Delta z=2 \mathrm{~m}$ from the surface to about $400 \mathrm{~m}$ depth. Deeper, the vertical resolution decreases, with $\Delta z \sim 60 \mathrm{~m}$ at the bottom. This choice was made to observe the vertical structure of the surface dynamics at high resolution. The stretching of the vertical levels can be seen in Fig. 1(right). Different horizontal resolutions were tested: $\Delta x=10,5,2,1$, and $0.5 \mathrm{~km}$.

The primary destabilization of the eddy (studied in section 3.2) depends relatively little on the horizontal resolution, since it is observed in all simulations for the range of values of 
$\Delta x$ considered. The diagnosis performed to analyze this primary instability were all done on the $\Delta x=1 \mathrm{~km}$ simulation. Secondary instabilities are observed for the simulations with $\Delta x=2,1$ and $0.5 \mathrm{~km}$. However, the time of apparition and the size of the small scale features (e.g the submesoscale vortices) differ in these simulations. To ensure that the model correctly resolves the frontal processes occurring at $t>100$ days, and to fully resolve the $O(<10) \mathrm{km}$ structure, the diagnosis of the secondary instabilities (see section 3.3) are performed on the $\Delta x=0.5 \mathrm{~km}$ simulation. For this one, the $\frac{\Delta x}{\Delta z}$ ratio is close to $\frac{N}{f_{0}}$ near the surface, see Fig. 1(right). This avoids the presence of spurious gravity waves associated with intense frontal processes, and allows a better resolution of the forward energy cascade (Snyder et al., 1993; Nadiga, 2014; Ménesguen et al., 2018).

For the study of the primary instability (discussed in section 3.2), the eddy is disturbed using two different methods. In the first one, the only source of perturbation is the numerical noise, due to the round-off errors at grid scale. In the second configuration we add a random noise. This noise is not spatially symmetric to avoid the dominant growth of specific azimuthal modes. The noise is introduced as a perturbation in the initial density and velocity fields. We define the random $2 \mathrm{D}$ perturbation $P(x, y)$ as a sum of 5000 random Gaussian functions in the horizontal plane:

$$
P(x, y)=\sum_{i=0}^{5000} A_{i} e^{-\frac{\left(x-x_{0}^{i}\right)^{2}}{2 \sigma_{x, i}^{2}}-\frac{\left(y-y_{0}^{i}\right)^{2}}{2 \sigma_{y, i}^{2}}},
$$

where $A_{i}, x_{0}^{i}, y_{0}^{i}, \sigma_{x, i}$ and $\sigma_{y, i}$ are chosen randomly and independently for each $i$, with $-1<A_{i}<1 ; 50<x_{0}^{i}, y_{0}^{i}<450 \mathrm{~km}$; and $5<\sigma_{x, i}, \sigma_{y, i}<40 \mathrm{~km}$. Then, the initial density perturbation is computed as:

$$
\rho_{n}(x, y, z)=\rho_{n, 0} P(x, y) e^{z / h_{n}}
$$

with $\rho_{n, 0}=0.01 \mathrm{~kg} \mathrm{~m}^{-3}$ and $h_{n}=500 \mathrm{~m}$. The depth $h_{n}=500 \mathrm{~m}$ is chosen so that the vertical extent of the perturbation is similar to the vertical extent of the perturbation at $t=7$ days in the simulation without noise. The velocity perturbation is in cyclogeostrophic balance with the $\rho_{n}$ field. This avoids the radiation of gravity waves during the first days of the simulation. The values of $\rho_{n, 0}$ are chosen so that the domain integral of the random perturbation's kinetic 
energy $E_{k}^{\prime}$ is about 10 times larger than the kinetic energy of the perturbation at $t=7$ days in the simulation without noise. Thus, $E_{k}^{\prime}(t=7$ days, no noise $)=1.910^{7} \mathrm{~m}^{5} \mathrm{~s}^{-2}$ and $E_{k}^{\prime}(t=7$ days, noise $)=2910^{7} \mathrm{~m}^{5} \mathrm{~s}^{-2}$. Notice that the energy of the perturbation is very weak compared to the total kinetic energy in the domain, which is about $1.810^{11} \mathrm{~m}^{5} \mathrm{~s}^{-2}$ at the initialization.

\subsection{Definitions and tools for the study of the simulation}

In this section, we describe the diagnostics performed. They allow us to characterize the dynamical processes involved in the destabilization of the eddy.

\subsubsection{Different kind of instabilities}

The different instabilities which may occur in geophysical flow can be inferred from the Potential Vorticity structure. In the primitive equation framework, the relevant definition of Potential Vorticity is the Ertel Potential Vorticity (hereafter PV). It is materially conserved in the absence of friction or diapycnal processes (Hoskins et al., 1985; Vallis, 2017), and is defined as:

$$
Q=\omega \cdot \nabla b
$$

where $b=-g \frac{\rho}{\rho_{0}}$ is the buoyancy, and $\boldsymbol{\omega}$ is the $3 \mathrm{D}$ absolute vorticity of the flow, approximated here by

$$
\boldsymbol{\omega}=\left(-\partial_{z} v, \partial_{z} u, \zeta+f_{0}\right)
$$

with $\zeta=\left(\partial_{x} v-\partial_{y} u\right)$ its $z$-component (relative vorticity hereafter). In the following, we will use the fully developed expression of the PV:

$$
Q=\left(f_{0}+\zeta\right) \partial_{z} b-\left(\partial_{z} v\right)\left(\partial_{x} b\right)+\left(\partial_{z} u\right)\left(\partial_{y} b\right)
$$

An eddy with no vertical shear can be unstable with respect to barotropic (BT) instability. A necessary condition is that its radial gradient of $\mathrm{PV}, \partial_{r} Q$, changes sign on the horizontal (i.e within a layer of constant density). 
Also, an eddy can be unstable with respect to baroclinic (BC) instability. The necessary condition is that its radial gradient of $\mathrm{PV}, \partial_{r} Q$, changes sign on the vertical (i.e between different layers). This latter change of sign can occur in the fluid interior (Phillips-type BC instability). If isopycnals outcrop, surface density gradients generate PV sheets (Bretherton, 1966) which, in turn, induce sharp PV gradients localized at the surface (Morel et al., 2006). The change of sign of the PV gradient can thus also occur between the surface and the interior (Charney-type BC instability), or between two sheet-type PV anomalies, at the surface and at the bottom (Eady-type instability) (Hoskins et al., 1985). In the mixed layer, the conditions for BC instability are changed and the theory described above is rather unsatisfactory. We refer the reader to Young \& Chen (1995) for further description.

In geophysical flows, symmetric instability (SI) may also occur when the product of planetary vorticity and potential vorticity becomes negative $(f Q<0)$ (Fjørtoft, 1950; Hoskins, 1974; Bell, 2018). This is an overturning instability occurring in baroclinic currents, which results from an imbalance between the pressure gradients and the inertial forces (Thomas et al., 2013). It occurs where the dynamic is frontal and where the PV of the flow is negative (in the Northern hemisphere) because of the second/and/or the third term of its expression (eq. (2)). Following Thomas et al. (2013), a necessary condition for SI instability can be derived. First, we define the balanced Richardson number of the flow

$$
R i=\frac{f_{0}^{2} N^{2}}{|\nabla b|^{2}}
$$

and the critical Richardson number

$$
R i_{c}=\frac{f_{0}}{\zeta_{g}}
$$

where $\zeta_{g}$ is the absolute vorticity of the geostrophic flow such as

$$
\zeta_{g}=f_{0}+\left(\partial_{x} v_{g}-\partial_{y} u_{g}\right)
$$

where $u_{g}$ and $v_{g}$ are the zonal and meridional components of the geostrophic velocity in the simulation. PV of a geostrophic flow is negative when $R i<R i_{c}$. When this condition is met, SI can develop. 


\subsubsection{Energy transfer terms}

The nature of the instabilities occurring in the flow may be investigated by studying the energy transfers. We assume that the flow may be decomposed as $\boldsymbol{u}=\langle\boldsymbol{u}\rangle+\boldsymbol{u}^{\prime}$, where the brackets $\langle\cdot\rangle$ denotes a mean state and the prime $\cdot^{\prime}$ is the perturbation relative to this mean state. First, the transfer from the mean kinetic energy (MKE) to the kinetic energy of the perturbation (EKE) may be expressed as:

$$
(M K E \rightarrow E K E)=H R S+V R S
$$

where

$$
H R S=-\left\langle u^{\prime 2}\right\rangle \partial_{x}\langle u\rangle-\left\langle u^{\prime} v^{\prime}\right\rangle \partial_{y}\langle u\rangle-\left\langle v^{\prime 2}\right\rangle \partial_{y}\langle v\rangle-\left\langle u^{\prime} v^{\prime}\right\rangle \partial_{x}\langle v\rangle
$$

is the contribution from the Horizontal Reynolds Stress, and

$$
V R S=-\left\langle u^{\prime} w^{\prime}\right\rangle \partial_{z}\langle u\rangle-\left\langle v^{\prime} w^{\prime}\right\rangle \partial_{z}\langle v\rangle
$$

is the contribution of the Vertical Reynolds Stress. Note that eq. (6) is obtained by integrating over the whole domain. Thus, formally, there are transport terms at the boundaries of the domain. In our simulations, these terms are null. Second, the transfer from the Potential Energy of the perturbation to the EKE is the Vertical Buoyancy Flux:

$$
V B F=\left\langle w^{\prime} b^{\prime}\right\rangle
$$

The predominance of HRS, VRS, or $\operatorname{VBF}(>0)$ in a region indicates a BT, KelvinHelmoltz type, or a BC instability respectively. More details on this decomposition and applications to the case of instabilities in the path of the Gulf Stream can be found in Gula et al. $(2015,2016)$. In our study, we use this decomposition to study (1) the primary instability of the eddy (Fig. 7 and A.17), and (2) the secondary instabilities occurring in the fronts around the eddy (Fig. A.18 and A.19).

In the first case, the mean flow defined by the variables $\langle u, v, w, b\rangle$ is assumed to be the initial state of the eddy. This choice allows to study the transfers of energy from the axisymmetric eddy to the perturbation. The perturbations $u^{\prime}, v^{\prime}, w^{\prime}, b^{\prime}$ are computed with respect to this initial state. Then, all time averaged products $\left\langle\cdot^{\prime} \cdot^{\prime}\right\rangle$ are performed using a 


$$
c_{\phi}^{m}(t, r)=\partial_{t} \phi_{m}(t, r) .
$$

The kinetic energy of the perturbation is defined as:

$$
E_{k}^{\prime}(t)=\frac{1}{2} \iiint d x d y d z\left(u_{r}^{\prime 2}+u_{\theta}^{\prime 2}\right)
$$


where $u_{r}^{\prime}$ and $u_{\theta}^{\prime}$ are the radial and azimuthal velocity perturbations. Similarly, the kinetic energy of the mode $m$ perturbation is:

$$
E_{k}^{\prime m}(t)=\frac{1}{2} \iiint d x d y d z\left(\left(u_{r}^{\prime m}\right)^{2}+\left(u_{\theta}^{\prime m}\right)^{2}\right) .
$$

In these two expressions, the integrals are performed over the whole domain of the simulation. Using exponential notation, time evolution of normal mode perturbation velocity reads:

$$
\mathbf{u}^{\prime m}(t)=\mathbf{u}_{0}^{\prime m} e^{i m(\theta-c t)}
$$

which yields an exponential form for the the energy growth:

$$
E_{k}^{\prime m}(t)=E_{k, 0}^{\prime m} e^{2 \sigma t}
$$

Where $E_{k, 0}^{\prime m}$ is the initial perturbation and $\sigma=R e(-i m c)$ is the growth rate. In our case, we choose to set $E_{k, 0}^{\prime m}=E_{k}^{\prime}(t=7$ days $)$. This reference is chosen as close as possible from the beginning of the simulation, without having problem with division by zero when computing the growth time. In the simulations, perturbation growth rates are inferred from the amplification factor $(\lambda)$ :

$$
\lambda_{E_{k}^{\prime m}}=\frac{E_{k}^{\prime m}(t)}{E_{k, 0}^{\prime m}} .
$$

The growth time is defined as $\tau_{E_{k}^{\prime m}}=\sigma_{E_{k}^{\prime m}}^{-1}$ with

$$
\sigma_{E_{k}^{\prime m}}=\frac{1}{2 t} \ln \left(\lambda_{E_{k}^{\prime m}}\right)
$$

Then,

$$
\tau_{E_{k}^{\prime m}}(t)=-2 t \ln \left(\frac{E_{k}^{\prime m}(t)}{E_{k, 0}^{\prime m}}\right) .
$$

When $\tau_{E_{k}^{\prime m}}$ is small, the growth of the perturbation is rapid.

\subsubsection{Critical level computation}

The position of the critical level for azimuthal mode 2 at the surface is found by seeking the position where the phase speed of the mode 2 perturbations is equal to the angular 
velocity of the eddy. This latter is here an azimuthal average of the azimuthal velocity $u_{\theta}$ of the eddy. At each time we thus obtain the position of the critical level for the mode 2 . We then average this position during the period $t=[50: 70]$ days, to obtain the typical position of the critical level during the linear phase of the perturbation.

The energy of normal modes can concentrate within the critical level, as further described in Nguyen et al. (2012) or Riedinger \& Gilbert (2014). In our study, we show that the presence of the critical level for mode 2 induces an intense stretching of the flow $S$. The latter is defined as the sum of the strain and the shear:

$$
S=\underbrace{\partial_{x} u-\partial_{y} v}_{\text {Strain }}+\underbrace{\partial_{x} v+\partial_{y} u}_{\text {Shear }} .
$$

It describes the tendency of the water parcels to be deformed by the ambient flow. Buoyancy gradients are steepened by this stretching. To describe the evolution of buoyancy gradients, we use the frontogenesis function $F$ associated with the buoyancy. It is calculated using its standard definition as follows (Hoskins, 1982):

$$
F(\mathbf{u}, b)=\partial_{x} u\left(\partial_{x} b\right)^{2}+\left(\partial_{x} v+\partial_{y} u\right) \partial_{x} b \partial_{y} b+\partial_{y} v\left(\partial_{y} b\right)^{2}
$$

The opposite of $F$ indicates the tendency of the buoyancy gradients to steepen:

$$
-F(\mathbf{u}, b)=\frac{d}{d t}|\nabla b|^{2}
$$

\section{Results}

\subsection{Overall course of the simulation}

We present here the overall course of the simulation, and we separate the different stages of the eddy evolution that we will discuss in the following sections.

Fig. 3 shows the evolution of relative vorticity after 70, 100, 180, and 300 days of simulation (panels (a), (b), (c), and (d) respectively). During the first part of the simulation (for $0<t<100$ days), a mixed BT/BC instability causes the eddy core to deform as spiral arms grow around it, see Fig. 3(a). Azimuthal mode 2 dominates, and the growth rate of the perturbation is roughly constant. The $\mathrm{BC}$ component of the instability leads to an intense 

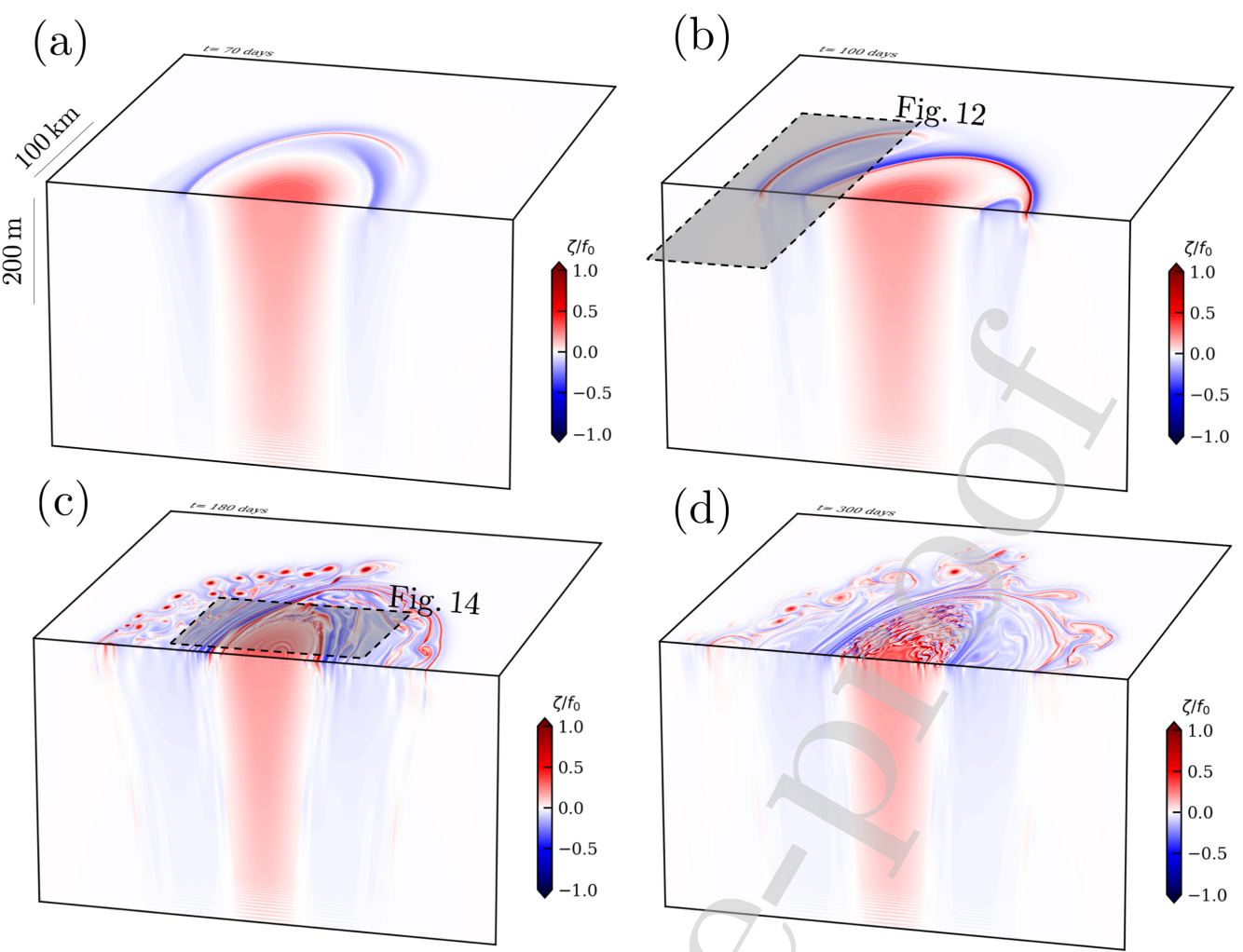

Figure 3: Snapshots of the normalized relative vorticity $\zeta / f_{0}$ at $t=70,100,180$ and 300 days in the $\Delta x=$ $0.5 \mathrm{~km}$ resolution simulation. Notice that the z-scale is very stretched to show the depth extension of the near-surface dynamics, see horizontal and vertical scalebars in panel (a). Gray rectangles in (b) and (c) show the regions studied in Fig. 12 and 14.

frontogenesis at the edge of the eddy and in the spiral arms, see Fig. 3(b). Then, secondary instabilities occur in the surface mixed layer, see Fig. 3(c). In particular, shear instability develops in the spiral arms, generating series of small vortices of $O(10) \mathrm{km}$. The steepening of buoyancy gradients at the edge of the eddy also induces diapycnal mixing. This causes PV to change sign and yields SI. The latter erodes the edge of the eddy by creating fine scale structures of $O(2) \mathrm{km}$ which are then ejected far away from the eddy, see Fig. 3(d). While secondary instabilities occur, the deepest part of the eddy $(z<-50 \mathrm{~m})$ remains a mesoscale tripole. It remains coherent during the last 6 months of the simulation. In subsection 3.2, we describe the evolution of the cyclone from $t=0$ to $t \sim 100$ days. Then, we present the secondary instabilities which occur in the surface mixed layer in subsection 3.3. 


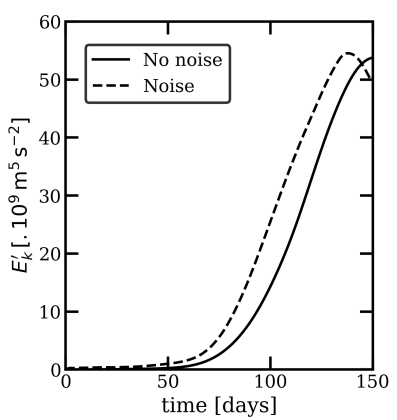

\subsubsection{Growth of normal modes}

\subsection{Primary instability: the monopole becomes a tripole}
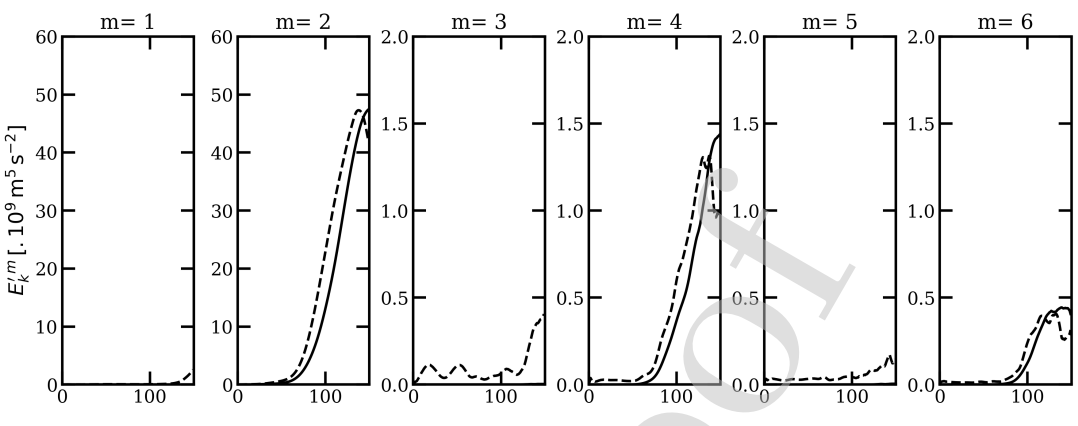

Figure 4: Time evolution of the perturbation kinetic energy $E_{k}^{\prime}$, for the simulation with random noise (dashed line) and without noise (solid line). The left panel shows the total kinetic/energy of the perturbation (for all modes). The panels on the right show the kinetic energy of the first six azimuthal normal modes. Notice that the vertical scale for the perturbation energy is not the same for plots corresponding to $m>2$.

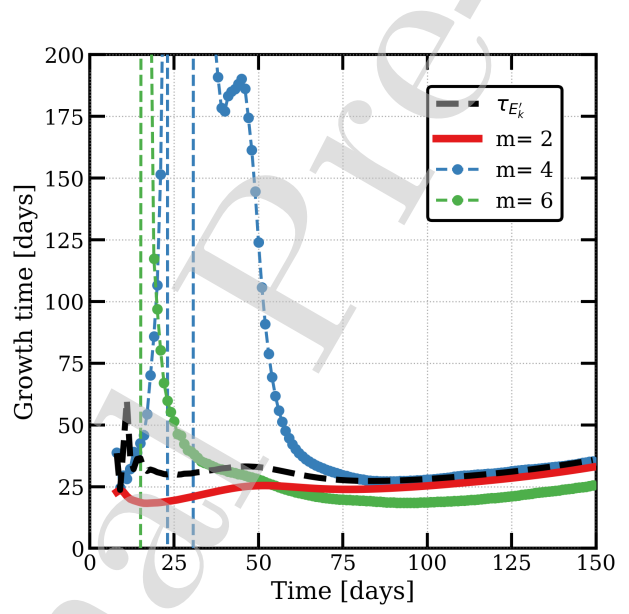

Figure 5: Time evolution of the growth time (in days) of the total kinetic energy of the perturbation $\tau_{E_{k}^{\prime}}$ (dashed line); and evolution of the growth time of the normal modes $\tau_{E_{k}^{\prime m}}$ for mode 2 (red), 4 (blue) and 6 (green), for the simulation without noise.

During the first 100 days of the simulation, the eddy destabilizes, with a domination of even azimuthal modes. To study the evolution of the perturbation, we compute its total kinetic energy at all times following eq. (13), and for each azimuthal mode following eq. (14), see (Fig. 4). The simulation without initial noise is compared to the simulation with a random noise. 
No difference is discernible in terms of growth of the perturbation between the two cases, showing that the structure is intrinsically unstable regardless of the initial perturbations. The total kinetic energy of the perturbation increases slowly at the first days of simulations. It then grows exponentially after $t \sim 50$ days, and tends to a plateau at $t \sim 150$ days.

The even modes have similar growth rates in the two simulations. In particular, the azimuthal mode 2 dominates, with values of energy close to the total energy of the perturbation. In the simulation where initial conditions are initially perturbed by a random noise, odd azimuthal modes are present at the initialization. These modes do not grow during the primary stage of the instability, meaning that they are stable. The processes involved in the primary destabilization of the eddy are well captured by the simulation without noise. In the following, we focus on the simulations performed without random noise.

The growth time of the perturbation is computed using eq. (19) for the total kinetic energy of the perturbation, for modes 2,4 and 6 (Fig. 5). The growth time of the total kinetic energy of the perturbation (i.e for all modes) is, on average, around 30 days. From the beginning of the simulation, the growth time of mode 2 is almost equal to the growth time of the total perturbation, confirming that the instability is controlled by mode 2 . Its growth time is roughly constant between day 50 and day 90, indicating that during this stage, mode 2 growth is exponential and evolves independently of the other modes (Gent \& McWilliams, 1986).

At the beginning of the simulation, transient perturbations occur in the core of the eddy, mostly stemming from the initial adjustment of the eddy. They are evanescent and rapidly decay (Riedinger \& Gilbert, 2014). However, during the first 20 days, their energy has similar magnitude to that of the unstable mode 2 . They are believed to trigger mode 2 . We next focus on the evolution of mode 2 since it dominates the instability. One can mention that for higher modes, nonlinear interactions most likely occur; their growth rates are not constant and have a similar evolution between each other.

\subsubsection{Instability mechanism}

The radial $\mathrm{PV}$ gradient $\partial_{r} Q$ is shown in Fig. 6. The horizontal change of sign of $\partial_{r} Q$ in the whole water column indicates that the structure may be barotropically unstable. 


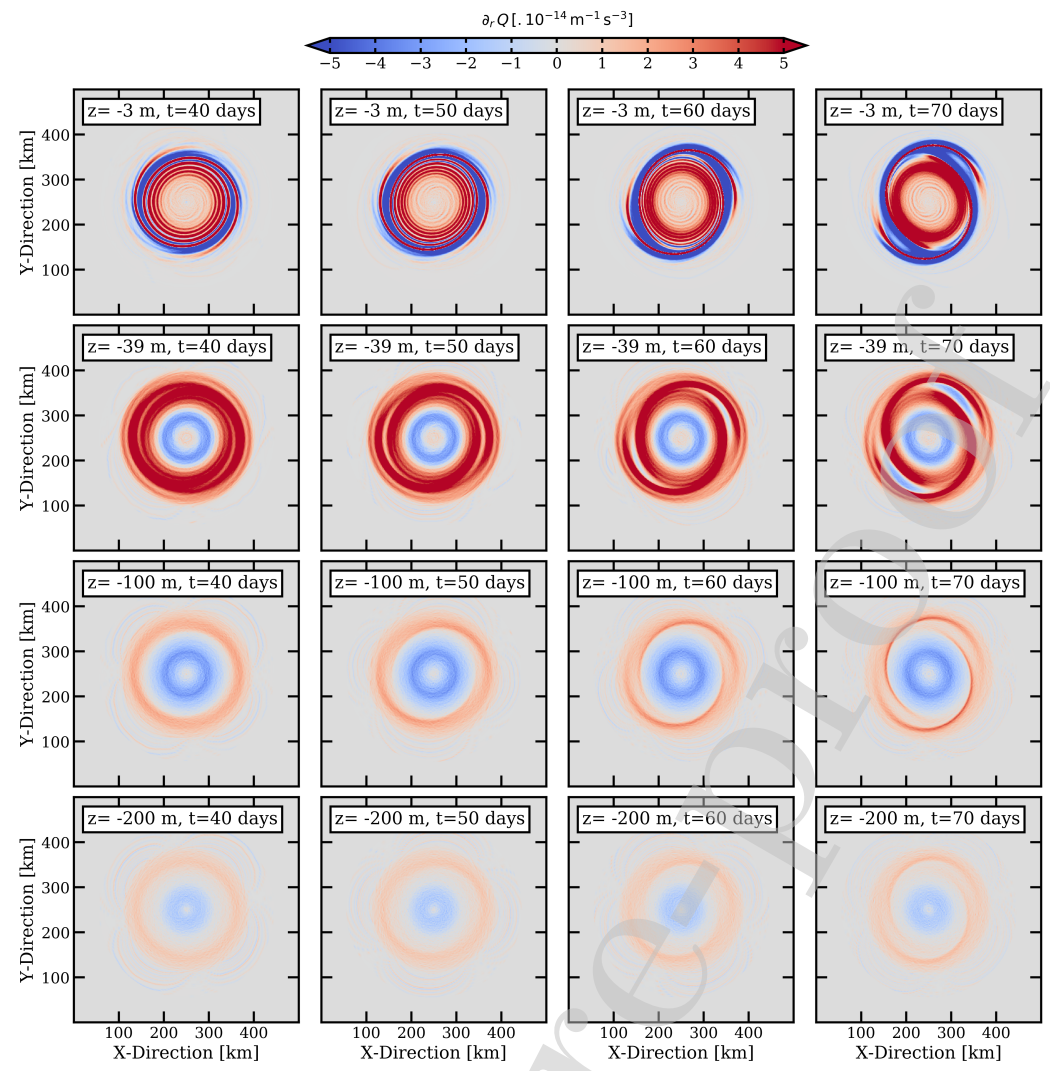

Figure 6: Radial derivative of the PV at $t=40,50,60$, and 70 days (from left to right), at 3, 39, 100 and $200 \mathrm{~m}$ depth (from top to bottom).

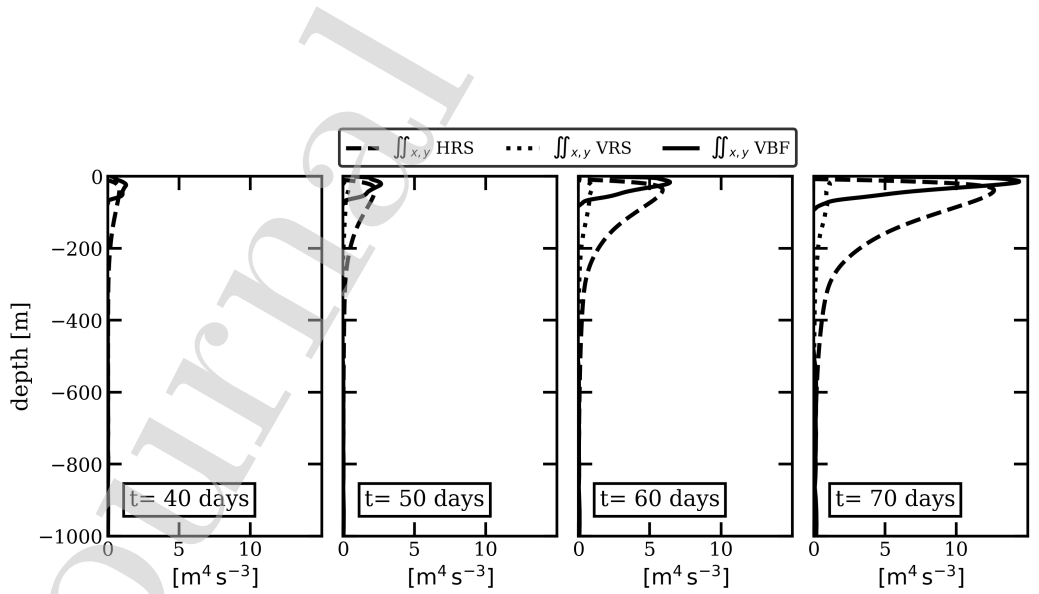

Figure 7: Profiles of energy transfer terms integrated on the horizontal at $t=40,50,60$, and 70 days (from left to right). HRS, VRS, and VBF stand for Horizontal Reynolds Stress, Vertical Reynolds Stress, and Vertical Buoyancy Flux. 


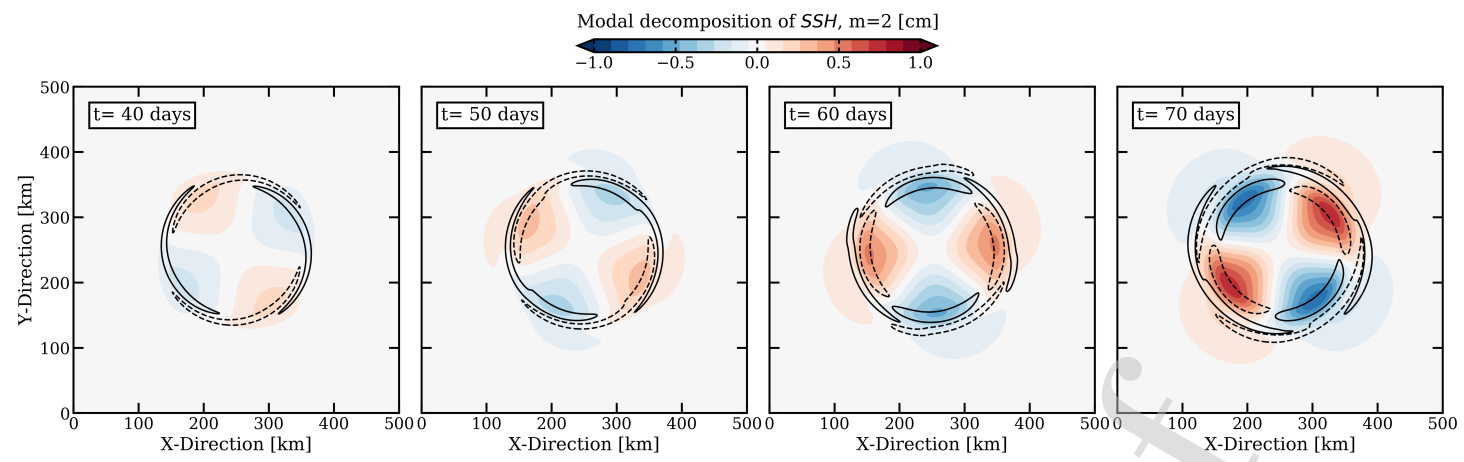

Figure 8: Mode 2 of the SSH during the linear phase of the perturbation, at $t=40,50,60$, and 70 days. It shows the counter-clockwise rotation of the eddy Rossby Wave (VRW) around the eddy. This wave rotates of about a quarter turn in 30 days. We superposed the contours $( \pm 0.02)$ of the mode 2 perturbation of normalized relative vorticity at the surface $\zeta^{\prime m=2} / f_{0}$.

At the center of the eddy, isopycnals outcrop (not shown), thus creating regions of high PV. Intersection lines between the surface and outcropping isopycnals are places of strong horizontal PV gradients. It can be seen in Fig. 6 at $z=-3 \mathrm{~m}$. This leads to a change of sign of $\partial_{r} Q$ in the vertical direction, between the surface and the interior PV gradients: the necessary condition for a Charney-type BC instability. The general stability conditions described by Pedlosky (1964); Eliassen (1983); Hoskins et al. (1985); Ripa (1991), stating that

$$
\left(u_{\theta}-\alpha\right) \partial_{r} Q<0
$$

and

$$
N^{2}\left(\delta z_{\rho}\right)^{2}>\left(u_{\theta}-\alpha\right)^{2}
$$

in the whole domain, for any $\alpha$, with $\delta z_{\rho}$ the vertical displacement of isopycnals, are also not respected in the whole domain (not shown). All necessary conditions for BT or BC instabilities to occur are thus met. Such mixed BT/BC instabilities have already been observed in high resolution numerical simulations, in particular in the case of Meddy-like eddies, see for instance Ménesguen et al. (2018).

Here, while the instability is mainly BT, it is strengthened by a small baroclinic mode near the surface. The energy transfer terms (HRS, VRS and VBF) integrated along the 


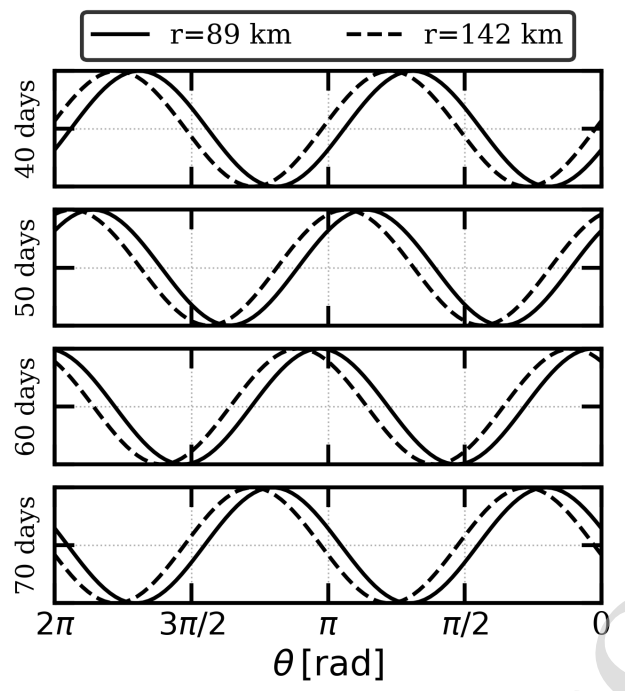

Figure 9: Comparison between the phase of the outer VRW and the inner VRW, in the mode 2 component of the SSH. Solid (resp. dashed) black lines shows cosinus with the phase of the mode 2 VRW at $\mathrm{r}=89 \mathrm{~km}$ (resp. $142 \mathrm{~km}$ ) for the same times as in Fig. 8 (from top to bottom).

horizontal directions are shown in Fig. 7. During the growth of the instability, HRS and VBF dominate, confirming that the instability is both BT and BC as predicted by the structure of the PV profile (Fig. 6). The HRS term is deep reaching and shows that the BT instability dominates the formation of the tripole. This instability penetrates deeply, and drives the formation of the tripole in the whole water column during the first 100 days of the simulation. The structure of the tripole at depth can be seen in Fig. 3(d), with a strong barotropic component of the positive and negative poles, and an outer ring of positive vorticity (i.e. the spiral arms). The VBF term is maximum near the surface, at the interface between the SML and the interior. This suggests that the instability is intensified by a BC component at the surface. Maps of vertically integrated transfer terms are presented in Appendix A, in Fig. A.17. In summary, together Fig. 7 and A.17 show that the BC instability participates in forming intense spiral arms in the SML, while the BT instability leads to the deformation of the core of the eddy.

To understand the destabilization of the eddy, one can notice that the propagation of the unstable mode 2 is that of a Vortex Rossby wave (VRW), rotating around the eddy. This wave is supported by the radial gradient of PV of the eddy. This process is similar to planetary Rossby waves in the ocean, supported by the meridional PV gradient induced 
by the $\beta$-effect. The signature of the VRW in the simulation can be seen in the mode 2 component of the SSH. The VRW rotates about a quarter turn in 30 days (Fig. 8) giving $c_{V R W}=0.05 \mathrm{rad}_{\text {days }}{ }^{-1}$. This value is consistent with the estimation of the phase speed of a baroclinic perturbation rotating around a unstable barotropic eddy in the quasi-geostrophic framework (see Appendix B). The mode 2 VRW exhibits different patterns depending on its position from the center of the eddy, see Fig. 9. It can be separated into two VRW rotating around the eddy at the same speed: an outer VRW (oVRW) at $r>100 \mathrm{~km}$, and an inner VRW (iVRW) at $r<100 \mathrm{~km}$. They are phase shifted. The oVRW is in advance compared to the iVRW. As these two waves interact, they amplify mutually, and the total perturbation rotating around the eddy grows (Flierl, 1988).

\subsubsection{Critical level consequences}

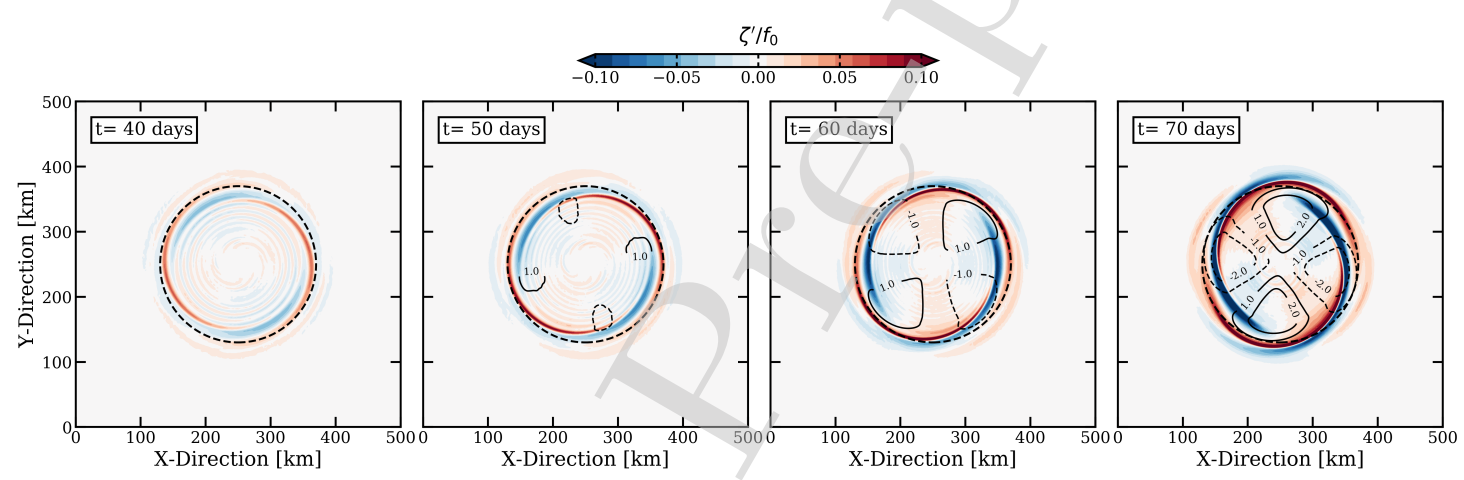

Figure 10: Time evolution of the perturbation of relative vorticity at the surface, at $t=40,50,60$, and 70 days. Contours of the mode 2 component of the surface radial velocity $u_{r}^{\prime m=2}$ for the values $-2,-1,1$, and 2 $\mathrm{km} \mathrm{day}^{-1}$ are superposed. It shows that the perturbation of the radial velocity is nearly in phase quadrature with the vorticity. The position of the critical level for the mode 2 is shown with the dashed black circle, at $r=120 \mathrm{~km}$.

The presence of a critical level (CL) for mode 2 leads to an important frontogenesis at the edge of the eddy and in the spiral arms. As discussed in Nguyen et al. (2012), the azimuthal speed of the VRW which carry the perturbation is small compared to the maximal swirl angular velocity of the eddy. The critical level is thus located far from the center of the eddy, where its swirl yelocity decreases outward (here on average at $r=120 \mathrm{~km}$ ). This value was obtained during the linear stage, following the method described in section 2.3.4. At the initial stage of the instability, for $t \sim 40-60$ days, the perturbation is wrapped around 

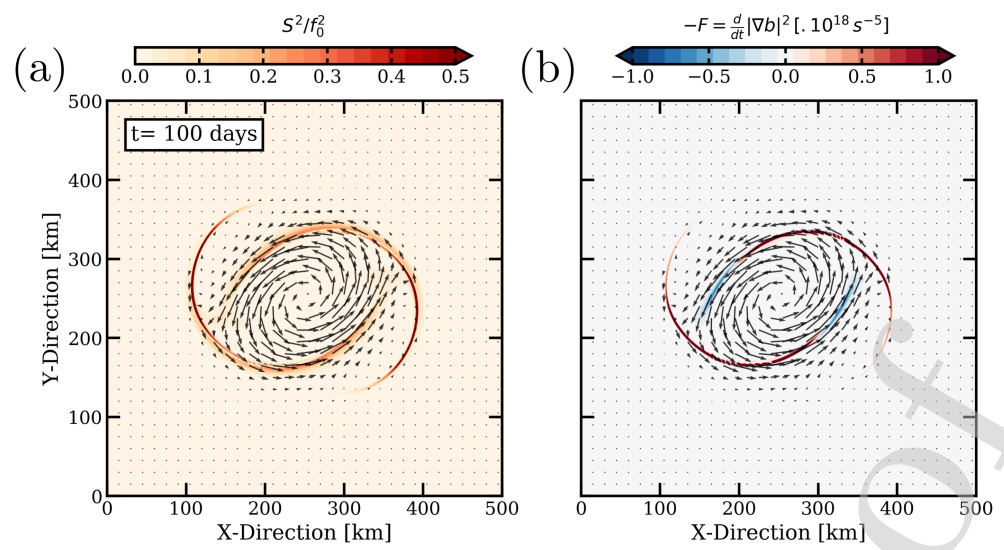

Figure 11: Frontogenesis mechanism at $t=100$ days. (a) Stretching at the surface normalized by the Coriolis frequency, and (b) opposite of the Frontogenesis function $(-F)$ at the surface, reflecting the steepening of buoyancy gradients. The horizontal velocity at the surface is superposed in both panels.

the eddy in the form of long vorticity filaments near the critical level position (Fig. 10). At this time, the eddy is still mostly circular, since the radial velocity is weak. Then, the mode 2 component of radial velocity $u_{r}^{\prime}$ grows. It reaches a value of several kilometer per day (see Fig. 10). Since it is nearly in phase quadrature with the vorticity, this radial velocity perturbation leads to the ellipticization of the eddy, and to the creation of spiral arms around the eddy.

At $t \sim 100$ days, after the linear stage of the mode 2 perturbation, the eddy becomes elliptical, and two spiral arms form. These arms are very thin since they were initially elongated around the CL. Stretching dominates the flow at the edge of the eddy and in the spiral arms (Fig. $11(\mathrm{a})$ ). The flow generated by the primary instability thus forces the gradients to steepen, in particular, the buoyancy gradients. The frontogenesis function $F$ associated with the buoyancy is computed at the surface and it is shown in Fig. 11(b). In the spiral arms and at the edges of the eddy, $-F$ is large. It reflects the steepening of the buoyancy gradients with time. Thus, the flow follows a frontal dynamic in these areas: the isopycnals outcrop and are very steep. Furthermore, the dynamical Rossby number $\epsilon=\zeta / f_{0}$ becomes locally larger than 1 (see Fig. 3), indicating that the circulation is mostly ageostrophic in these regions (Stegner \& Dritschel, 2000). These observations are in agreement with the results of Nguyen et al. (2012) and Hua et al. (2013), who show that 
density gradients are enhanced at critical levels.

\subsection{Secondary instabilities and their impact on small scale structures}

The primary BT/BC instability saturates at $t \sim 120$ days, after the initial monopole has evolved into a stable tripole; this tripole remains stable during the next 6 months of the simulation below the surface mixed layer. Inside the surface mixed layer, the intense frontogenesis leads to sharp fronts near the surface.

The small scale structures located in the surface mixed layer follow a Surface Quasi Geostrophic (SQG) dynamic. Because they have a small horizontal size, these surface features have a small vertical influence. Indeed, in the framework of the SQG model, following Isern-Fontanet et al. (2008), the streamfunction is $\Psi_{S Q G} \propto e^{\frac{N_{0} k}{f_{0}} z}$, where $k$ is the horizontal wavenumber at the surface. The smaller horizontal scales decay in the vertical faster than larger ones. The vertical extension of surface structures scales as:

$$
L_{z}=\frac{f_{0} L}{2 \pi N_{0} f_{0}}
$$

where $L$ is the horizontal size of surface features and $N_{0} \sim 10^{-2} \mathrm{~s}^{-1}$ the typical stratification, leading to

$$
L_{z} \sim 10^{-3} L
$$

In our case, filaments and submesoscale vortices which evolve at the surface have a size of $O(<10) \mathrm{km}$. Therefore, they have a vertical influence over $O(<10) \mathrm{m}$. This vertical scale applies to the stratified fluid below the surface mixed layer. It shows that the submesoscale is largely confined to the mixed layer, as it can be seen in Fig. 3 at $t \geq 100$ days.

\subsubsection{Barotropic instability at the surface as a source of submesoscale}

In the spiral arms, the steep PV gradients create favorable conditions for BT instability. BT instability occurs in a thin surface mixed layer in which the flow is confined, and leads to the destabilization of the spiral arms at $t \sim 110$ days. A mean PV section of a spiral arm (Fig. 12(b)) was computed by averaging 10 vertical sections at $t=110$ days (Fig. 12(a)). The PV anomaly of the spiral arm is confined in the upper $10 \mathrm{~m}$. The cross-filament derivative 

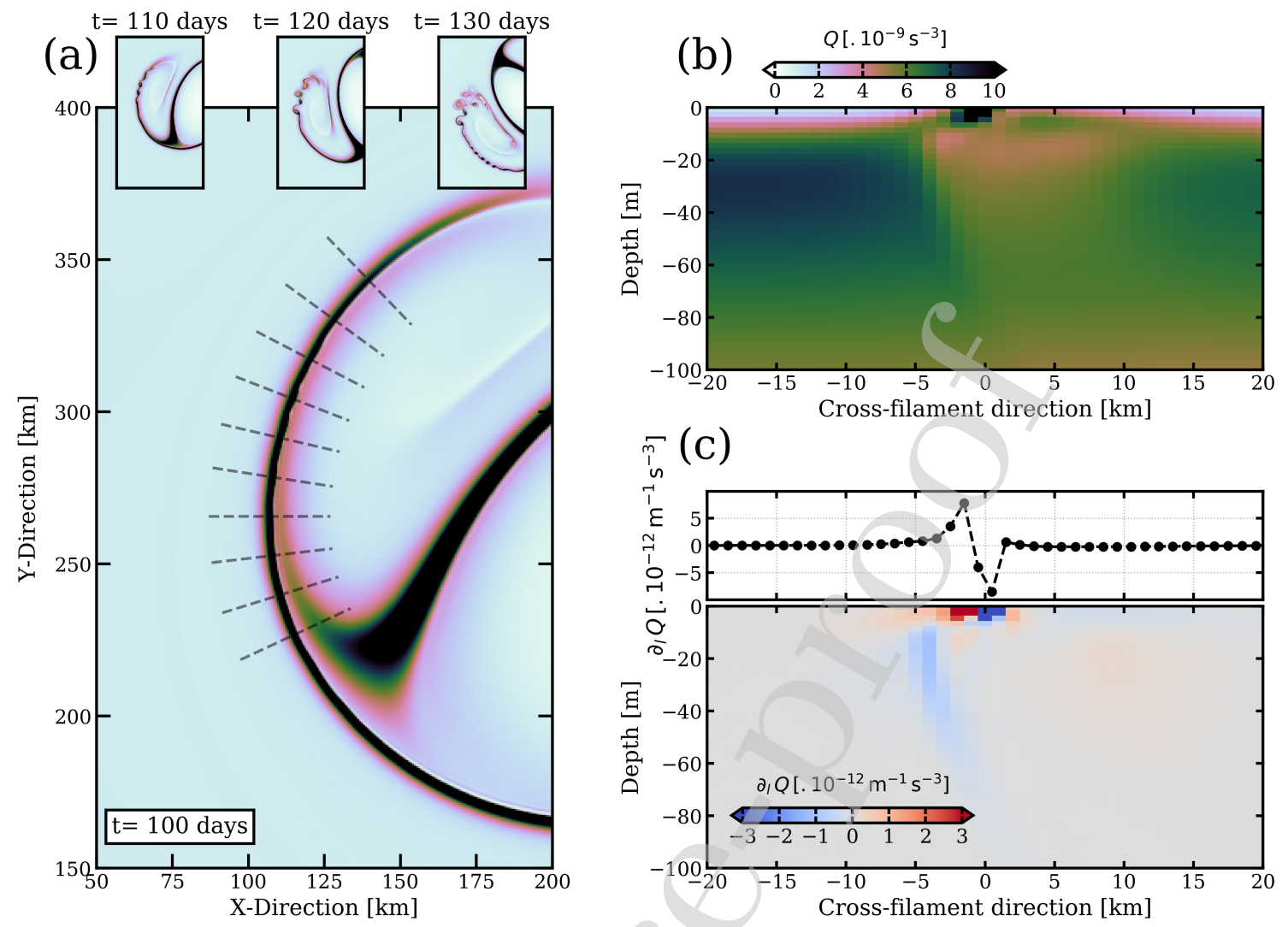

Figure 12: (a) Snapshot of surface PV, at $t=100$ days before the BT instability in the spiral arms. The domain shown is the western part of the eddy. Inserts present snapshots of surface PV at $t=110,120$ and 130 days. (b) Mean vertical section of PV computed using 10 sections perpendicular to the spiral arm at $t=100$ days (their position is indicated in (a) by the grey dashed lines). The colormap of (a) and (b) is the same. (c) Derivative of the PV along the axis of the section, at the surface (top), and in the first 100 meters (bottom).

of the PV $\left(\partial_{l} Q\right)$ changes sign along the section, signature of BT instability (Fig. 12(c)). Small ambient perturbations trigger instability, forming a row of small vortices of positive relative vorticity. The first ones appear at $t \sim 110$ days, at the tip of the arms. At $t \sim 130$ days, the whole arms destabilize (Fig. 12(a)), and evolve into a row of submesoscale vortices surrounding the main eddy (Fig. 3(c)). A few of the satellite vortices remain coherent for about 6 months. The energy transfer terms in the spiral arm are shown in Fig. A.18, see the calculation method in section 2.3.2. It shows a strong signature of positive HRS in the arm when it becomes unstable, confirming that destabilization is due to a purely BT instability in the surface mixed layer. These observations show that BT instability of the spiral arms of 
a large unstable eddy is an efficient mechanism for the formation of submesoscale vortices.

To investigate the possibility of secondary instability to be triggered by grid-scale numerical noise, a sensitivity test was performed, adding a random noise in the simulation at $t=$ 90 days. In this case, the arms destabilize slightly earlier, at $t=105$ days, but in a similar way, still forming a row of small vortices. As for the primary instability of the vortex, the vorticity arms are intrinsically unstable.

At the edge of the eddy core, the cross-front PV gradient changes sign on the horizontal as well as on the vertical (not shown). Thus, the edge of the eddy is unstable with respect to a mixed BT/BC instability. Because the PV gradients are weaker, growth rates are slower than observed in the arms. However, at $t \sim 150$ days, the small vortices generated around the eddy start to merge with the vortex edge, triggering instability. This is confirmed by large values of HRS and VBF at the edge, where the small eddies interact with the larger one (Fig. A.19). Then, a short wavelength elliptical VRW propagates around the eddy (see the deformation of the eddy's edge in Fig. 3(c).

\subsubsection{Evidence of symmetric instability at the edge of the eddy}

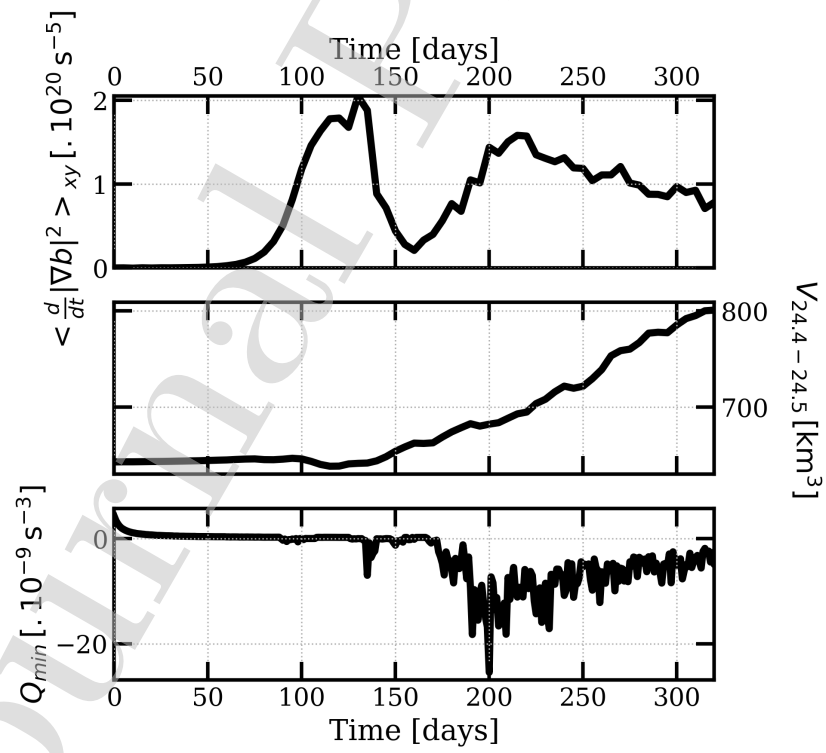

Figure 13: Time evolution of (top) the average of $-F(\mathbf{u}, b)=\frac{d}{d t}|\nabla b|^{2}$ at the surface, (middle) the volume between the $\rho=1024.4 \mathrm{~kg} \mathrm{~m}^{-3}$ and the $\rho=1024.5 \mathrm{~kg} \mathrm{~m}^{-3}$ isopycnals, and (bottom) the minimum of PV in the domain. 


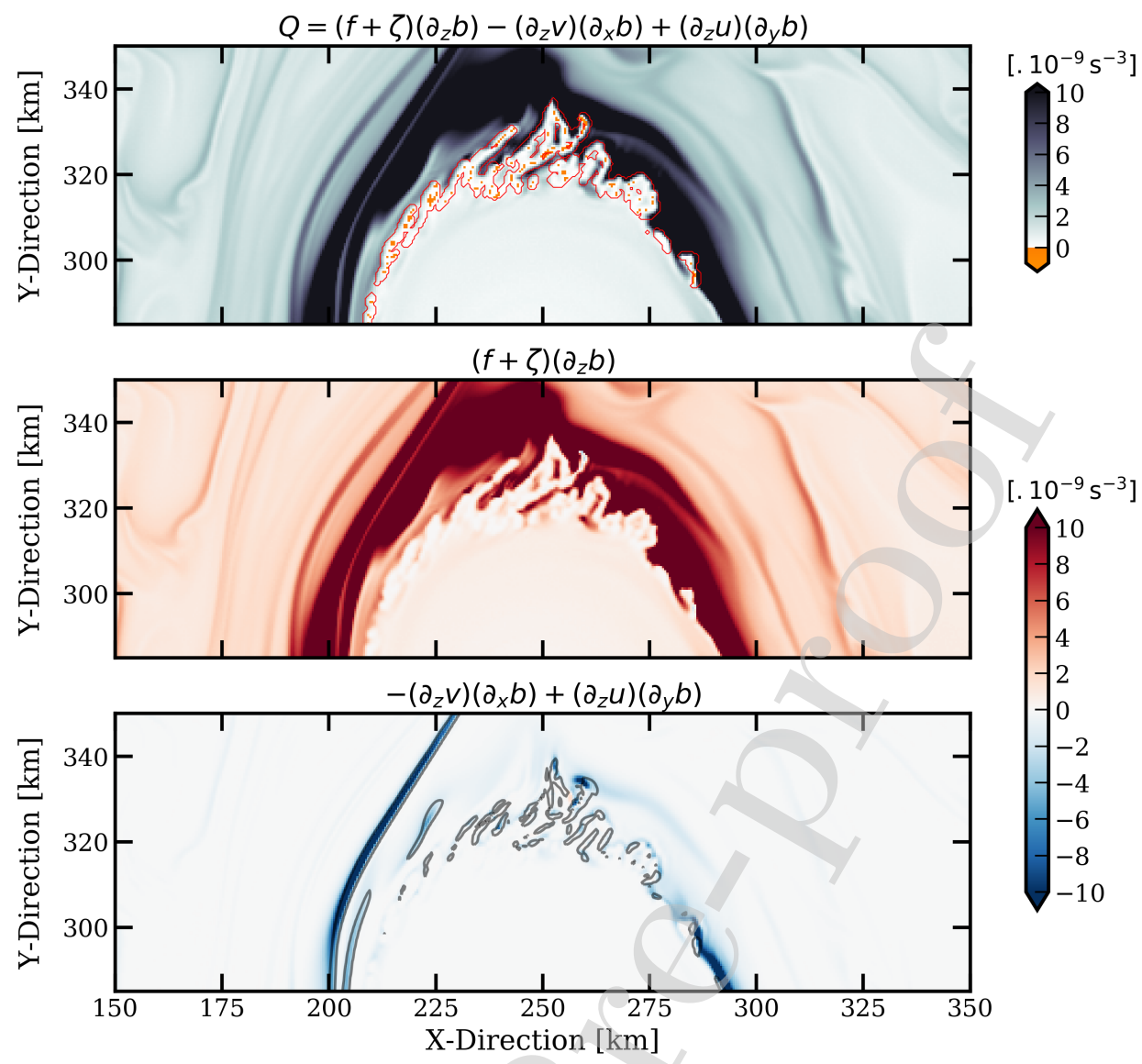

Figure 14: Decomposition of the PV at $t=180$ days, at the southern part of the eddy. (top) PV at the surface; the yellow indicates places where the PV is negative, and the red lines indicate regions where the criterion for SI to occur $R i<R i_{c}$ is respected. (middle) First term of the PV. (bottom) Second and third terms of the $\mathrm{PV}$; contours of $-F=10^{-18} \mathrm{~s}^{-5}$ are superposed as grey thin lines.

A second phase of frontogenesis is triggered as the small scale VRW deforms the front at the edge of the eddy through intense stretching. Time evolution of the mean $-F$ at the surface is shown in Fig. 13(top). At $t=160$ days, steepening of the buoyancy gradient starts to increase for a second time. The second and third terms of the PV (in eq. (2)) become very intense locally, see e.g. Fig. 14(bottom). Indeed, the variations of velocity are localized at the surface, and variations of buoyancy occur at scales of $O(1) \mathrm{km}$. These terms are negative and of the same order of magnitude as the first term of the PV, see Fig. 14(middle), leading to a PV close to zero where the fronts are sharp (Fig. 14(top)).

The horizontal buoyancy variations occur at very fine scales (see the contours of $-F$ at $t=180$ days on Fig. 14(bottom)). The circulation at the edge of the eddy becomes 
strongly ageostrophic from $t \sim 160$ days, leading to diapycnal mixing at the edge of the eddy. The latter acts in decreasing stratification, leading to a PV sign change. In our model, since diapycnal mixing is parameterized through the KPP-scheme, we do not attempt to characterize it, and rather focus our attention on the dynamical processes resulting of the diapycnal mixing.

A first explanation for the decrease of the PV due to diapycnal mixing can be found by introducing changes of buoyancy in the expression of the PV. We present in Appendix C how the PV sign can change if it is initially close to zero.

A second explanation can be found in the integral formulation of the impermeability theorem derived in Haynes \& McIntyre (1990) and discussed in Morel \& McWilliams (2001):

$$
\frac{d}{d t} \iiint_{\Omega} d \Omega \Delta Q=-f \frac{d \Omega}{d t},
$$

where $\Omega$ is a domain with periodic boundary conditions or with no normal flow (our case here), $d \Omega$ a volume element, $\Delta Q$ the $\mathrm{PV}$ anomaly with respect to the $\mathrm{PV}$ background, and $\frac{d \Omega}{d t}$ the volume variations of the layer. This formulation states that the bulk integral of the PV anomaly can only be changed by a loss or a gain of the volume inside a layer comprised between two isopycnals. At $t \sim 120$ days, the frontogenesis at the edge of the eddy has led to an isopycnal steepening at the surface. In particular, the layer between the $\rho=1024.4 \mathrm{~kg} \mathrm{~m}^{-3}$ and the $\rho=1024.5 \mathrm{~kg} \mathrm{~m}^{-3}$ isopycnals is thin, and slopes very steeply towards the surface. This specific layer is chosen because it represents isopycnals outcropping at the edge of the eddy. Then, after the destabilization of the eddy edge and diapycnal mixing, this layer has become thicker, and the front is less steep, see Appendix D, Fig. D.20. Following eq. (21), this change of thickness leads to the decrease of the bulk integral of the PV, which explains the decrease of local PV, and the formation of negative PV. In Fig. 13, we present the time evolution of this layer volume. It is fairly consistent with the appearance of negative PV.

The presence of locally negative PV, along with an examination of the Richardson number show that the edge of the eddy is subject to SI. The area where $R i<R i_{c}$ is indicated in Fig. 14(top). In this area, the PV of the geostrophic flow is negative. When the PV changes sign, due to the second and third terms in eq. (2), SI develops. From $t \sim 160$ days, the edge of the eddy thus becomes strongly deformed by vorticity changes over scales of $O(1) \mathrm{km}$. SI 
then propagates inward. At the end of the simulation, at $t=300$ days, the SI pattern is present in the whole core of the eddy, at the surface, see Fig. 3(d).

\section{Sensitivity study}

We have performed other simulations to study the sensitivity of our results to the numerical setup. Hereafter, the 'reference simulation' refers to the simulation on the $f$-plane, with a cyclonic eddy, $\Delta x=1 \mathrm{~km}, 256$ vertical levels, and the numerical settings discussed in section 2.2. All the sensitivity tests are performed by varying one or several of these parameters. The details of these simulations are not shown in this paper, but we mention their most salient features.

Firstly, we changed the numerical settings of the model. Changing the horizontal advection scheme to a third-order upwind advection scheme (UP3) leads to a larger implicit viscosity, and the fronts at the surface are less intense and become unstable later than in the reference run. Fewer small vortices result from the instability and they are less intense. If we remove the KPP-scheme parameterization in the interior, an important noise appears at $t \sim 30$ days because of a lack of vertical turbulent closure. The sensitivity on the boundary conditions has been tested by running the model in a larger domain $(1000 \times 2000 \mathrm{~km})$ with the eddy initialized at $\left(x_{0}, y_{0}\right)=(400,300) \mathrm{km}$. This does not affect the dynamics of the eddy.

Secondly, we performed the reference simulation with a flat bottom at $4000 \mathrm{~m}$ with the same number and stretching of vertical levels. This does not affect the primary instabilities (the growth of mode 2 and the formation of row of small vortices), showing that a bottom at $1500 \mathrm{~m}$ is sufficient to describe the dynamic of the eddy. However, the SI occurs 30 days earlier. This is due to the fact that the spacing between the vertical levels is larger than in the reference run. The $\Delta x / \Delta z$ ratio is not close enough to $N / f_{0}$, and the frontal dynamic is not well resolved.

Thirdly, we tested the cyclone-anticyclone asymmetry. We initialized the opposite composite density anomaly and recalculated the temperature and the velocity fields associated with it. This produces a warm core anticyclonic eddy. We put this structure in the same stratification as for the reference simulation, and we ran the model. We observed a different 
evolution than for the cyclonic case. At $t=100$ days, the anticyclone was still stable, and its horizontal shape was similar to its initial shape. An azimuthal mode 2 then grew, as in the cyclonic case. It led to the formation of a tripole with spiral arms at $t \sim 280$ days. Then, the arms also became unstable and formed submesoscale vortices of negative relative vorticity. For the anticyclone, the growth of the perturbation was much slower than for the cyclone. Furthermore, the two satellites in the formed tripole were smaller than in the reference simulation. This asymmetry can be explained as follows. Considering the cyclogeostrophic balance, a cyclone and an anticyclone cannot have both antisymmetric velocity and density anomalies. Here, we initialized the anticyclone with the density anomaly. Thus, its swirling velocity was smaller than that of the cyclone, and the initial anticyclone had a weaker kinetic energy. In Stegner \& Dritschel (2000), a comparison between the shape of tripoles obtained from the destabilization of a cyclone and an anticyclone can be seen in their Fig. 7.

Finally, we performed the reference simulation, but now on a $\beta$-plane, with $\beta=2.110^{-11} \mathrm{~m}^{-1} \mathrm{~s}^{-1}$ (the mean value of $\beta$ in the area of the composite eddy). The primary instability of the eddy strongly differs from the $f$-plane simulations. After 20 days of simulation, the eddy starts drifting northwestward, with a trailing Rossby wave wake. The evolution of the eddy is similar to that shown in Fig. 3 of McWilliams et al. (1986). Then, mode 2 grows. At $t \sim 70$ days, two asymmetric arms are present around the initial eddy. They become unstable similarly as in the reference simulation, creating submesoscale vortices around the initial eddy.

\section{Discussion}

\subsection{On the stability of the composite eddy}

In this paper, we studied the stability of a composite cyclone as revealed by Argo floats in the northern Arabian Sea. With this simulation, we were able to study numerous kinds of instabilities, thanks to very high horizontal and vertical resolutions.

First, we observed that a deep azimuthal mode 2 barotropic instability deforms the eddy, which eventually evolves into a tripole after about 4 months of simulation. This kind of instability has already been observed for specific 2D analytical barotropic shielded vortices (Carton \& McWilliams, 1989; Stegner \& Dritschel, 2000), 3D meddy-like eddies (Yim \& 
Billant, 2015; Meunier et al., 2015; Ménesguen et al., 2018), or surface eddies (Meunier et al., 2019). We show that in the vicinity of a realistic eddy intensified at the surface, azimuthal mode 2 perturbations is the main driver for its deformation.

Second, we showed that a baroclinic instability together with the presence of a critical level for the most unstable mode is the main cause for the generation of a highly ageostrophic flow around the eddy. This generates sharp fronts in the surface mixed layer where the Rossby number is large $(\epsilon>1)$.

Third, these fronts become unstable to a barotropic instability in the surface mixed layer. This leads to the generation of submesoscale cyclones and filaments. This highlights the predominance of secondary instabilities in the evolution of isolated eddies.

Finally, our results indicate that symmetric instability may be ubiquitous in large oceanic vortices, even cyclonic ones. Indeed, for small $B u$ vortices, the critical level is located near the edge of the eddy (Nguyen et al., 2012) where energy is concentrated. This leads to the formation of intense fronts. Near these fronts, a strongly imbalanced circulation occurs, and yields diapycnal mixing. As previously observed in the context of idealized simulations (Nadiga, 2014; Brannigan et al., 2017), this causes PV to change sign locally, and SI develops at the edge of the cyclonic eddy. This happens when the edge of the eddy becomes unstable due to the interaction between submesoscale vortices and the large eddy. It highlights the fact that in the real ocean the interactions between small scale features and large mesoscale eddies may play a key role in the stability of the latter.

Previous studies considering analytical vortices showed that when a vortex is unstable, it may break into smaller vortices, depending on its initial profile (Carton et al., 1989; Stegner \& Dritschel, 2000). A major result of this study is that even if instabilities occur, the composite eddy is not destroyed/and remains as a large scale coherent structure for the last 6 months of the simulation, similar to specific kind of analytical vortices (Hua et al., 2013; Ménesguen et al., 2018). The composite re-stabilizes into a tripolar structure for which the positive vorticity pole is slightly elliptical but coherent. 


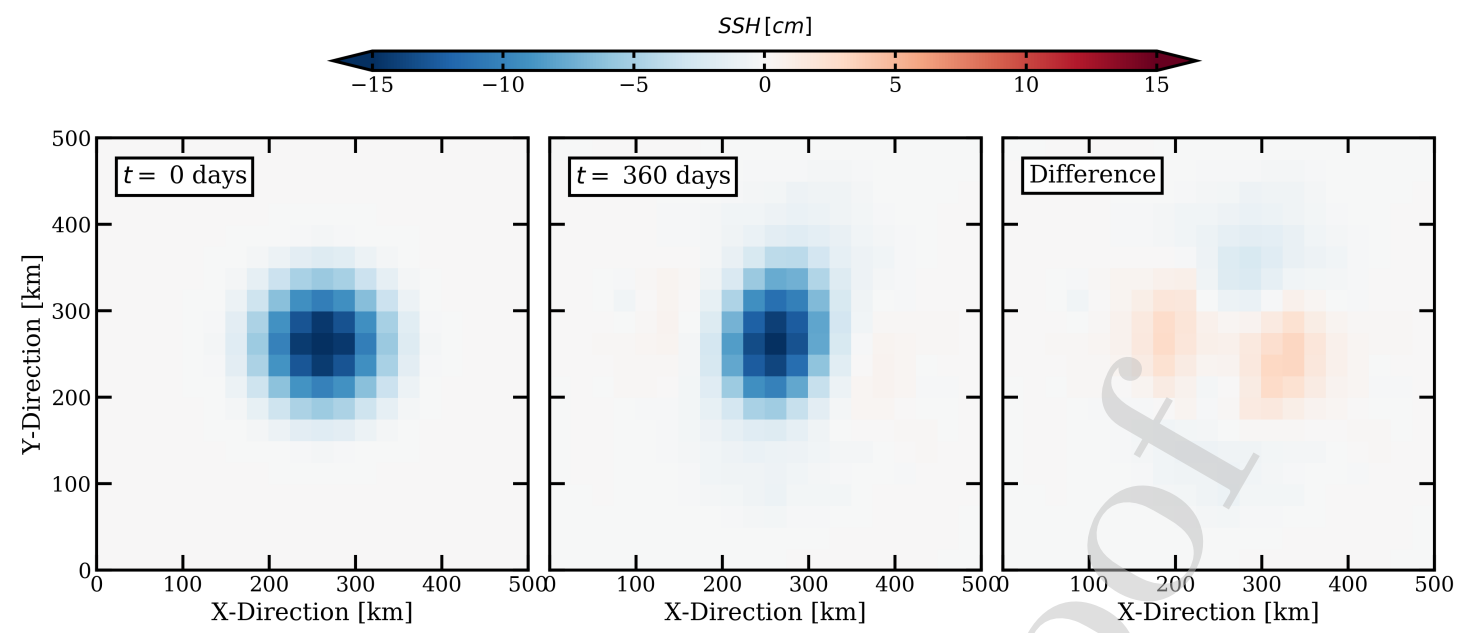

Figure 15: SSH signature of the eddy at initialization (left), at the end of the simulation ( $t=360$ days) (middle), and the difference between the two. The resolution of the plot is downgraded to the same resolution as the altimetric product used to generate the composite eddy.

\subsection{On the detectability of eddy instabilities}

To infer the detectability in altimeter products of the series of instabilities described in this paper, we computed the SSH signature of the eddy at the beginning and at the end of the simulation, using a downgraded resolution similar to the one of the altimetric product (Fig. 15). The signature of the tripole at the end of the simulation is that of an elliptical cyclonic eddy, of ellipticity $e=0.72$. The difference between the tripole and the initial cyclone is very weak, with variations of SSH of $O(1) \mathrm{cm}$, which is of the order of magnitude of the formal error induced by the spatio-temporal interpolation of altimetric products (see Appendix A in de Marez et al. (2019)). This indicates that the tripolar structures often observed in numerical studies may be present in the ocean but may not be observed due to the coarse resolution of the altimetry.

The mean ellipticity of eddies used to compute the composite was on average 0.75 . The cyclone at the end of the simulation thus have an ellipticity comparable with the ellipticity of eddies observed in altimetric products, which were used to compute the composite. Since the composite was computed assuming axisymetry, it is of interest to assess the impact of this hypothesis on the final state of the modelled eddy, by computing an azimuthal average (Fig. 16). The differences between the initialization and the final form act over scale of $O(10) \mathrm{km}$ on the horizontal and $O(10) \mathrm{m}$ on the vertical. Considering the very coarse resolution of the 

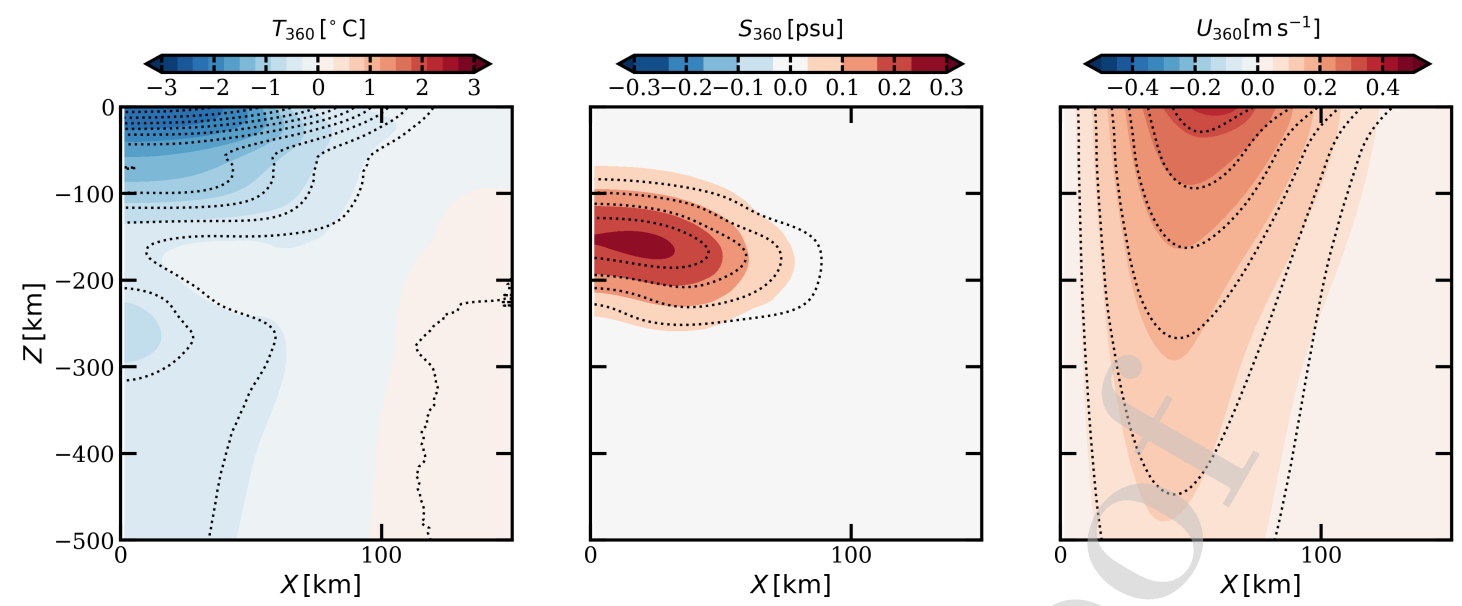

Figure 16: Comparison between the azimuthal average of the final form of the eddy (at $t=360$ days) and the composite. Color contours are azimuthal averages of the final eddy while black dashed contours indicate the same contours for the initial eddy.

dataset available to study the 3D structure of eddies $(e . g O(10) \mathrm{km}$ for the altimetric product and $O(10) \mathrm{m}$ for Argo floats), and the error implied by the composite method $O(1-5) \mathrm{km}$ (Chaigneau et al., 2011), these differences can not be seen with in situ data.

The small scale features we observe during the secondary instabilities can be observed qualitatively on snapshots of true color (Lévy et al., 2018) or of sea surface temperature (Buckingham et al., 2017). However, no altimetric product allowing to predict the dynamical quantities at this resolution are currently available. This means that neither the primary instability nor the secondary instabilities can be clearly captured in a systematic way using the currently available altimetric and floats data. Considering SSH only, the composite eddy we studied evolves little, and fairly represents the eddy observed in the altimetry which can live for several months. 
636

\section{Appendix A. Maps of energy transfer terms}

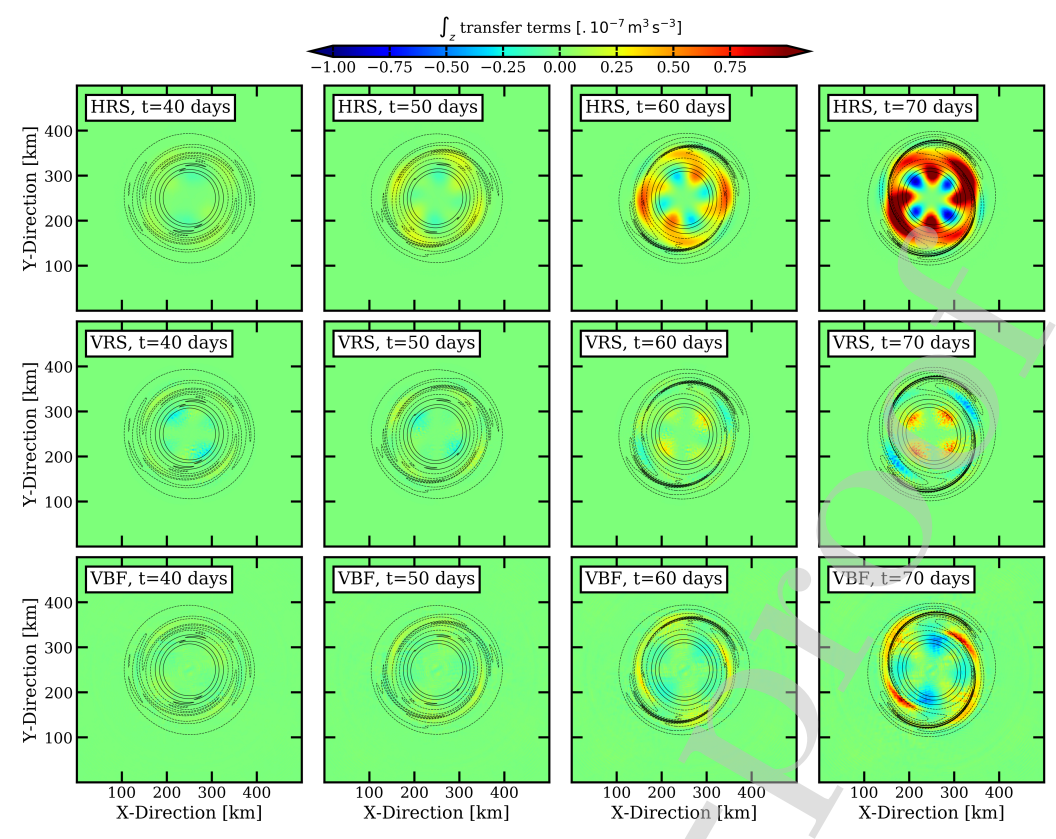

Figure A.17: Snapshots of energy transfer terms integrated on the vertical at $t=40,50,60$, and 70 days (from left to right). HRS, VRS, and VBF stand for Horizontal Reynolds Stress, Vertical Reynolds Stress, and Vertical Buoyancy Flux. 
$\int_{z}$ transfer terms $\left[\cdot 10^{-7} \mathrm{~m}^{3} \mathrm{~s}^{-3}\right]$

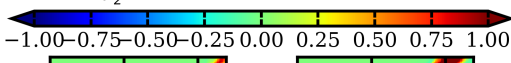

HRS, $\mathrm{t}=105.0$ days HRS, $\mathrm{t}=110.0$ days HRS, $\mathrm{t}=120.0$ days HRS, $\mathrm{t}=130.0$ days
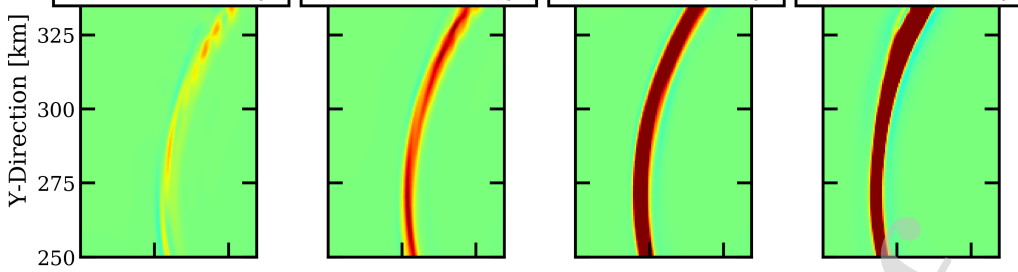

VRS, $t=105.0$ days
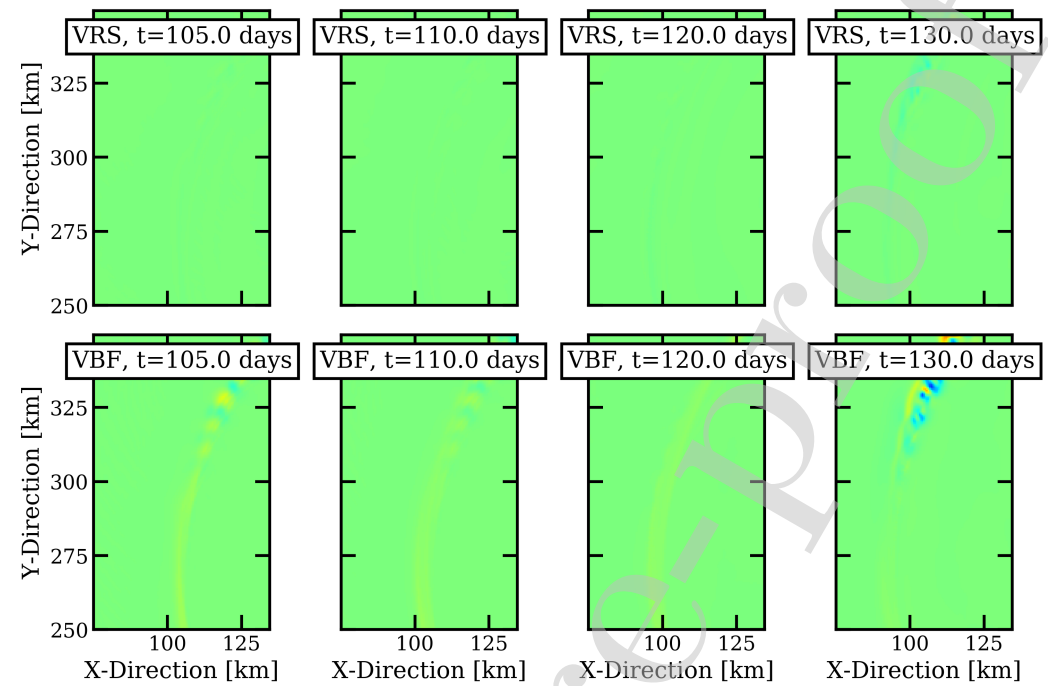

Figure A.18: Snapshots of energy transfer terms integrated on the vertical, zoom on the left spiral arm which becomes unstable, at $t=105,110,120$, and 130 days (from left to right). 


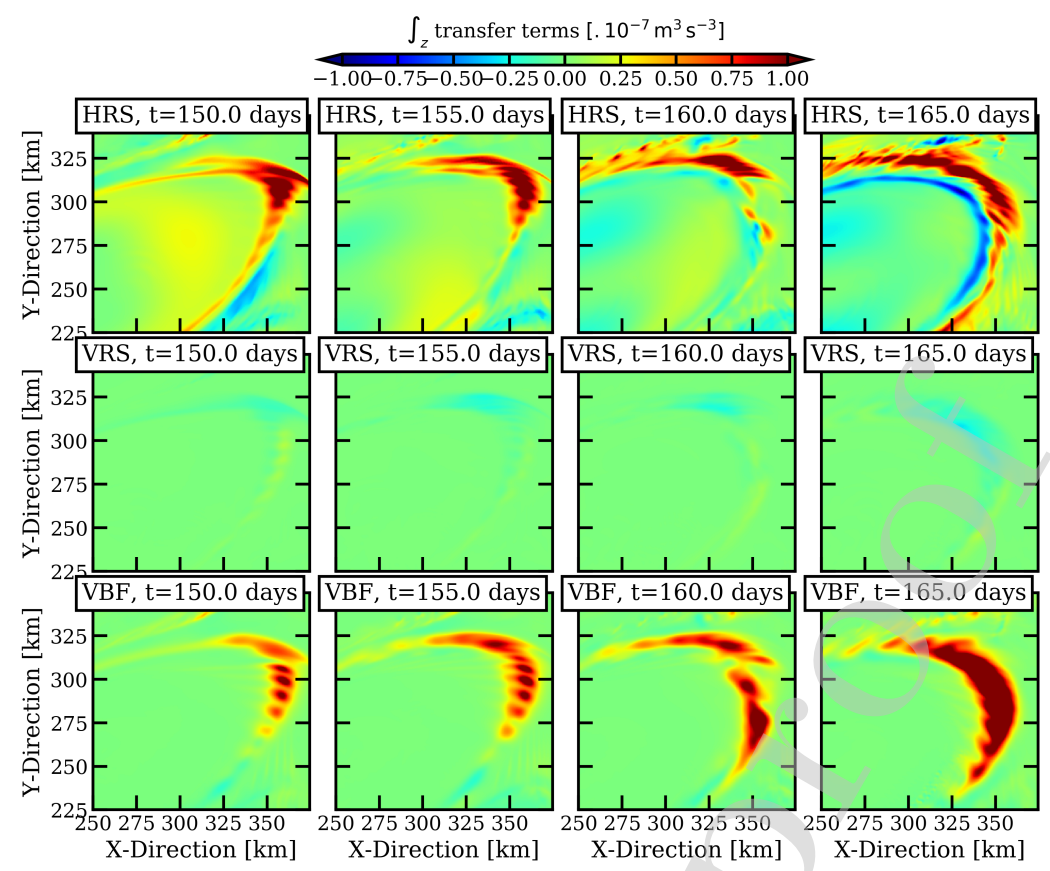

Figure A.19: Snapshots of energy transfer terms integrated on the vertical, zoom on the edge of the eddy which becomes unstable, at $t=150,155,160$, and 165 days (from left to right). 


\section{Appendix B. Estimating the phase velocity of the perturbation in the QG ap- proximation}

To estimate the value of the phase velocity of the mode 2 perturbation, one can use the framework of the continuously stratified quasi-geostrophic (QG) model (Vallis, 2017). We consider a mean state which is a circular barotropic eddy of azimuthal velocity $U_{\theta}$ and potential vorticity $\bar{q}$. We introduce a perturbation in the form of a normal mode, of potential vorticity $q^{\prime}$ and current function $\psi^{\prime}$. Linearizing the system around the mean state gives (Pedlosky, 2013):

$$
\left(U_{\theta}-c\right) q^{\prime}-\psi^{\prime} \bar{q}_{r}=0
$$

where $c$ is the azimuthal velocity of the perturbation and $\bar{q}_{r}$ is the radial derivative of $\bar{q}$. The potential vorticity of the perturbation is

$$
q^{\prime}=\Delta \psi^{\prime}+\frac{f_{0}^{2}}{N_{0}^{2}} \partial_{z} \psi^{\prime}=L \psi^{\prime}
$$

$N_{0}$ is the ambient stratification of the flow, which is in our case $\sim 10^{-2} \mathrm{~s}^{-1}$. Using (B.1) and (B.2), the azimuthal velocity of the perturbation can be expressed as:

$$
c=U_{\theta}-\frac{\psi^{\prime} \bar{q}_{r}}{L \psi^{\prime}}
$$

For our purpose, we only discuss the order of magnitude of the velocity of the perturbation, which is:

$$
c \sim U_{\theta}-\frac{\bar{q}_{r}}{\alpha}
$$

where $\alpha$ approximates the operator $L$ such as:

$$
\alpha=\underbrace{\frac{4 \pi^{2}}{R^{2}}+\left(\frac{m}{R}\right)^{2}}_{\alpha_{B T}}+\underbrace{\frac{f_{0}^{2}}{h N_{0}^{2}}}_{\alpha_{B C}},
$$

with $m$ the azimuthal mode of the perturbation, $R \sim 100 \mathrm{~km}$ the radius of the eddy and $h \sim 50 \mathrm{~m}$ the typical scale of variation of the perturbation. The first two terms $\left(\alpha_{B T}\right)$ 
represent the barotropic perturbation, while the third one $\left(\alpha_{B C}\right)$ corresponds to the vortex stretching induced by the baroclinicity of the flow.

In the case we study here, $\bar{q}_{r} \sim \frac{U_{\theta}^{\max }}{(R / 2)^{2}}$, with $U_{\theta}^{\max } \sim 0.5 \mathrm{~m} \mathrm{~s}^{-1}$ the value of $U_{\theta}$ at $\sim R / 2$. This gives $\bar{q}_{r} \sim 210^{-10} \mathrm{~m}^{-1} \mathrm{~s}^{-1}, \alpha_{B T} \sim 1.410^{-9} \mathrm{~m}^{-2}$ and $\alpha_{B C} \sim 510^{-9} \mathrm{~m}^{-2}$. At the edge of the eddy, $U_{\theta} \sim 0.1 \mathrm{~ms}^{-1}$. Using eq. (B.4) gives an estimation of the phase velocity $c \sim 0.06 \mathrm{~m} \mathrm{~s}^{-1}$. This corresponds to a velocity of $0.06 \mathrm{rad}_{\text {days }}{ }^{-1}$, which is in fairly good agreement with the phase velocity computed in the simulation, and seen in Fig. 8. Notice that this estimation of the phase velocity depends on the values chosen for each quantity, and does not take into account the baroclinicity of the mean flow. However, it shows that to obtain a proper estimation, we need to consider both the $\mathrm{BT}$ and the $\mathrm{BC}$ components of the perturbation. 


\section{Appendix C. How can diapycnal mixing change the sign of the PV ?}

As pointed in Morel \& McWilliams (2001), 'when mixing does occur, it is not easy to predict the change of the PV for a fluid parcel'. Using the Ertel form of the PV, we assess here how can diapycnal mixing change the sign of the PV.

In the case of a front localized at the edge of an axisymmetric eddy, with no radial velocity, one can write the PV in polar coordinates as:

$$
Q=\zeta_{a} \partial_{z} b+\left(\partial_{z} u_{\theta}\right)\left(\partial_{r} b\right),
$$

with $\zeta_{a}=\zeta+f_{0}$. If we consider the Thermal Wind Balance, this becomes

$$
Q=\zeta_{a} \partial_{z} b-f^{-1}\left(\partial_{r} b\right)^{2} .
$$

We study the variations of PV when the buoyancy is modified by diapycnal mixing. We introduce $\Delta_{z} b$ and $\Delta_{r} b$ the variations of buoyancy respectively along the $z$ and $r$ axis. The value of $\mathrm{Q}$ may thus be approximated by:

$$
Q=\frac{\Delta_{z} b}{\Delta z} \zeta_{a}-f^{-1}\left(\frac{\Delta_{r} b}{\Delta r}\right)^{2} .
$$

If diapycnal mixing occurs, the gradients of buoyancy diminish along the $z$-axis and/or the $r$-axis. In terms of variations of buoyancy, we thus have

$$
\left\{\begin{aligned}
\Delta_{z} b & \rightarrow \Delta_{z} b-b_{z} * \\
\Delta_{r} b & \rightarrow \Delta_{r} b-b_{r} *,
\end{aligned}\right.
$$

where $b_{r} *>0$ and $b_{z} *>0$ are the variations of buoyancy induced by the diapycnal mixing. They are positive since diapycnal mixing leads to a decrease of the variations of buoyancy. We assume here that during the time in which diapycnal mixing occurs, the flow does not vary, and $\zeta_{a}$ thus remains constant. This strong assumption is not true at long times, when the new circulation is adjusted, but it allows to keep the calculation and the reasoning simple.

We define $Q *$ as the PV value after the diapycnal mixing. Introducing the new buoyancy variations (C.2) in the expression of the PV (C.1) gives: 


$$
Q *=\underbrace{\frac{\Delta_{z} b}{\Delta z} \zeta_{a}-f^{-1}\left(\frac{\Delta_{r} b}{\Delta r}\right)^{2}}_{Q}-\frac{b_{z} *}{\Delta z} \zeta_{a}-f^{-1}\left(\frac{b_{r} *}{\Delta r}\right)^{2}+2 f^{-1} \frac{\Delta_{r} b b_{r} *}{(\Delta r)^{2}} .
$$

The two first terms on the right hand side are the PV before the diapycnal mixing. We then define $\Delta Q=Q *-Q$, the variation of $\mathrm{PV}$ during the diapycnal mixing. It is expressed such as:

$$
\Delta Q=\underbrace{-\frac{b_{z^{*}}}{\Delta z} \zeta_{a}}_{\Delta Q_{1}} \overbrace{-f^{-1}\left(\frac{b_{r} *}{\Delta r}\right)^{2}}^{\Delta Q_{2}}+\underbrace{2 f^{-1} \frac{\Delta_{r} b b_{r} *}{(\Delta r)^{2}}}_{\Delta Q_{3}}
$$

If the vorticity in the flow is positive, and if it is located in the northern hemisphere, $\zeta_{a}>0$. Thus, both $\Delta Q_{1}$ and $\Delta Q_{2}$ are strictly negative. The sign of $\Delta Q_{3}$ depends on the sign of $\Delta_{r} b$. In the case of a front leading to a positive velocity along the $\theta$-axis (e.g at the edge of a cyclone), the buoyancy gradients along the $r$-direction are positive. Then, $\Delta Q_{3}>0$ and the sign of $\Delta Q$ thus depends on the relative importance of its terms. The sign of $\Delta Q$ thus depends on the scale of variations and the values of the gradients. For instance, in a case where the mixing only occurs on the vertical, $b_{r} *=0$. The variation of $\mathrm{PV}$ thus reduces to $\Delta Q=\Delta Q_{1}<0$. In this case, the PV decreases. If before the mixing it was already close to 0, the decrease of the PV can thus lead to the generation of negative PV where the fluid has been mixed. A summary of the possible sign that $\Delta Q$ can take depending on the direction of the current, and the direction of the mixing is shown in Table C.1. 


\begin{tabular}{|c|c|c|c|c|}
\hline & $\Delta \mathrm{Q}_{\mathbf{1}}$ & $\Delta \mathrm{Q}_{\mathbf{2}}$ & $\Delta \mathrm{Q}_{\mathbf{3}}$ & $\Delta \mathrm{Q}$ \\
\hline $\mathrm{CE}$ & $<0$ & $<0$ & $>0$ & $?$ \\
\hline $\mathrm{AE}$ & $>0$ & $<0$ & $<0$ & $?$ \\
\hline $\mathrm{CE}$ vert. & $<0$ & $=0$ & $=0$ & $<0$ \\
\hline AE vert. & $>0$ & $=0$ & $=0$ & $>0$ \\
\hline CE horiz. & $=0$ & $<0$ & $>0$ & $?$ \\
\hline AE horiz. & $=0$ & $<0$ & $<0$ & $<0$ \\
\hline
\end{tabular}

Table C.1: Summary of the sign that can take $\Delta Q . \mathrm{CE}$ and AE designate cyclonic and anticyclonic flow; vert. and horiz. indicate cases where the mixing only occurs on the vertical direction or the horizontal direction; ? indicates that the sign of $\Delta Q$ depends on the relative importance of the different terms.

\section{Appendix D. Section of the front at the edge of the eddy}
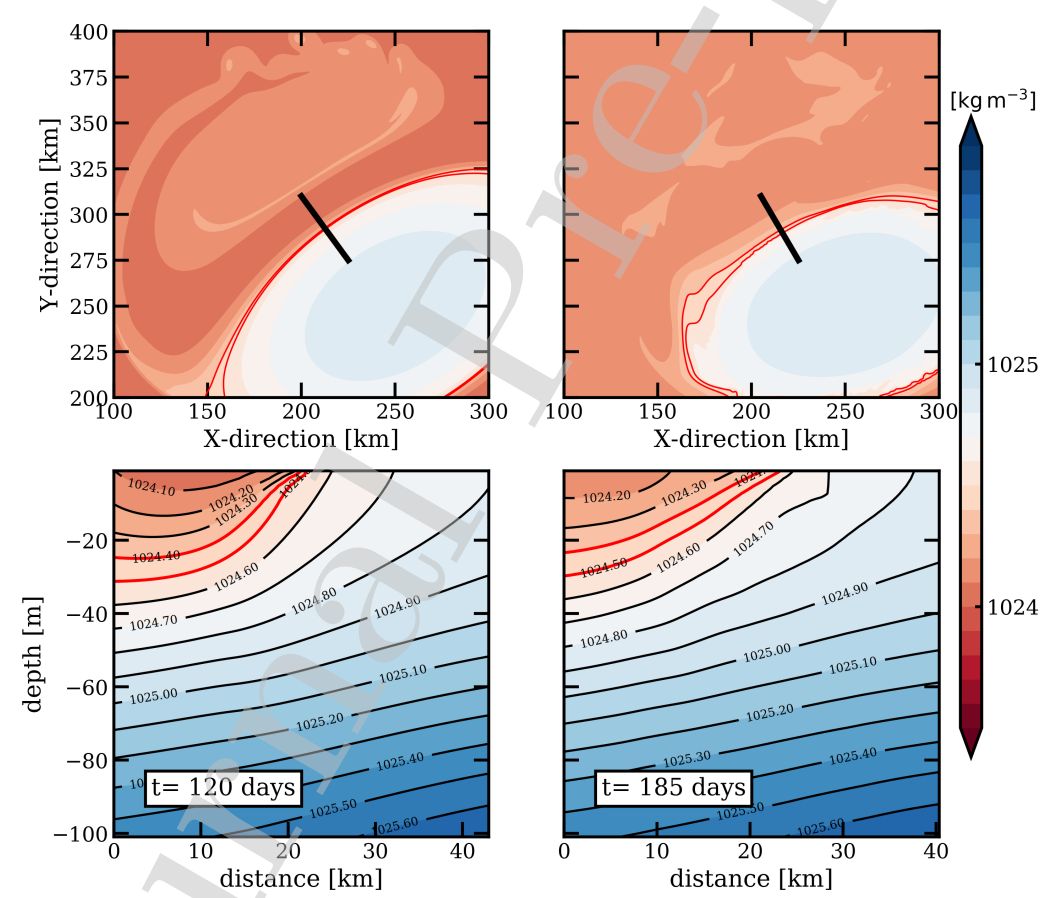

Figure D.20: (top) Snapshots of density at the surface at $t=120$ days (left) and $t=185$ days (right). (bottom) Vertical sections of density at $t=120$ days (left) and $t=185$ days (right), their position is shown by the black thick lines in the top panels. Red contours indicates the isopycnals surrounding the layer we discuss, for which we compute the volume shown in Fig. 13 
699

700

701

\section{Acknowledgments}

This work was funded by the Direction Générale de l'Armement (DGA) via a full grant for Charly de Marez's PhD. This work is also a contribution to PRC 1069 'Meso and submesoscale vortices in the Atlantic and Indian Ocean', funded by CNRS and RFBR. Simulations were performed using the HPC facilities DATARMOR of 'Pôle de Calcul Intensif pour la Mer' at Ifremer, Brest, France. The authors thank N. Lahaye for helpful discussions, and the two anonymous reviewers whose remarks improved the contents of this paper. Model output is available upon request. 


\section{References}

Al Saafani, M. A., Shenoi, S. S. C., Shankar, D., Aparna, M., Kurian, J., Durand, F., \& Vinayachandran, P. N. (2007). Westward movement of eddies into the Gulf of Aden from the Arabian Sea. Journal of Geophysical Research, 112, C11. doi:10.1029/2006JC004020.

Armi, L., Hebert, D., Oakey, N., Price, J. F., Richardson, P. L., Rossby, H. T., \& Ruddick, B. (1989). Two Years in the Life of a Mediterranean Salt Lens. Journal of Physical Oceanography, 19, 354-370. doi:10.1175/1520-0485(1989)019<0354:TYITLO>2 . 0.CO; 2.

Bell, M. J. (2018). Reduction of the linear stability problem for general zonal flows with any Rossby and Richardson numbers to a PDE. Quarterly Journal of the Royal Meteorological Society, 144, 2260-2276. doi:10.1002/qj.3352.

Brannigan, L., Marshall, D. P., Naveira Garabato, A. C., Nurser, A. J. G., \& Kaiser, J. (2017). Submesoscale Instabilities in Mesoscale Eddies. Journal of Physical Oceanography, 47, 3061-3085. doi:10.1175/JPO-D-16-0178.1.

Bretherton, F. P. (1966). Critical layer instability in baroclinic flows. Quarterly Journal of the Royal Meteorological Society, 92, 325-334. doi:10.1002/qj . 49709239302.

Buckingham, C. E., Khaleel, Z., Lazar, A., Martin, A. P., Allen, J. T., Naveira Garabato, A. C., Thompson, A. F., \& Vic, C. (2017). Testing Munk's hypothesis for submesoscale eddy generation using observations in the North Atlantic. Journal of Geophysical Research: Oceans, 122, 6725-6745. doi:10.1002/2017JC012910.

Carton, X., \& McWilliams, J. (1989). Barotropic and Baroclinic Instabilities of Axisymmetric Vortices in a Quasigeostrophic Model, . 50, 225-244. doi:10.1016/S0422-9894(08) 70188-0.

Carton, X. J., Flierl, G. R., \& Polvani, L. M. (1989). The Generation of Tripoles from Unstable Axisymmetric Isolated Vortex Structures. Europhysics Letters (EPL), 9, 339 344. 
Chaigneau, A., Le Texier, M., Eldin, G., Grados, C., \& Pizarro, O. (2011). Vertical structure of mesoscale eddies in the eastern South Pacific Ocean: A composite analysis from altimetry and Argo profiling floats. Journal of Geophysical Research, 116, C11. doi:10.1029/2011JC007134.

Chelton, D. B., deSzoeke, R. A., Schlax, M. G., El Naggar, K., \& Siwertz, N. (1998). Geographical Variability of the First Baroclinic Rossby Radius of Deformation. Journal of Physical Oceanography, 28, 433-460. doi:10.1175/1520-0485(1998) 028<0433: GVOTFB> 2.0. $\mathrm{CO} ; 2$.

Chelton, D. B., Gaube, P., Schlax, M. G., Early, J. J., \& Samelson, R. M. (2011). The Influence of Nonlinear Mesoscale Eddies on Near-Surface Oceanic Chlorophyll. Science, 334, 6054. doi:10.1126/science.1208897.

de Marez, C., L'Hégaret, P., Morvan, M., \& Carton, X. (2019). On the 3d structure of eddies in the Arabian Sea. Deep Sea Research Part I: Oceanographic Research Papers, 150, 103057. doi:10.1016/j.dsr.2019.06.003.

Dong, C., McWilliams, J. C., Liu, Y., \& Chen, D. (2014). Global heat and salt transports by eddy movement. Nature Communications, 5, 3294. doi:10.1038/ncomms4294.

Eliassen, A. (1983). The Charney -Stern Theorem on Barotropic-Baroclinic Instability, . (pp. 563-572). doi:10.1007/978-3-0348-6608-8_11.

Fjørtoft, R. (1950). Application of integral theorems in deriving criteria of stability for laminar flows and for the baroclinic circular vortex, .

Flierl, G. R. (1988). On the instability of geostrophic vortices. Journal of fluid mechanics, 197, 349-388. doi:10.1017/S0022112088003283.

Gent, P. R., \& McWilliams, J. C. (1986). The instability of barotropic circular vortices. Geophysical ES Astrophysical Fluid Dynamics, 35, 209-233. doi:10.1080/03091928608245893.

Gula, J., Molemaker, M. J., \& McWilliams, J. C. (2015). Gulf Stream Dynamics along the Southeastern U.S. Seaboard. Journal of Physical Oceanography, 45, 690-715. doi:10. 1175/JPO-D-14-0154.1. 
Gula, J., Molemaker, M. J., \& McWilliams, J. C. (2016). Topographic generation of submesoscale centrifugal instability and energy dissipation. Nature Communications, 7, 12811. doi:10.1038/ncomms12811.

Halo, I., Backeberg, B., Penven, P., Ansorge, I., Reason, C., \& Ullgren, J. (2014). Eddy properties in the Mozambique Channel: A comparison between observations and two numerical ocean circulation models. Deep Sea Research Part II: Topical Studies in Oceanography, 100, 38-53. doi:10.1016/j.dsr2.2013.10.015.

Haynes, P. H., \& McIntyre, M. E. (1990). On the Conservation and Impermeability Theorems for Potential Vorticity. Journal of the Atmospheric Sciences, 47, 2021-2031. doi:10.1175/ 1520-0469 (1990) 047<2021:OTCAIT>2.0.CO;2.

Hoskins, B. (1982). The mathematical theory of frontogenesis. Annual review of fluid mechanics, 14, 131-151. doi:10.1146/annurev.fl.14.010182.001023.

Hoskins, B. J. (1974). The role of potential vorticity in symmetric stability and instability. Quarterly Journal of the Royal Meteorological Society, 100, 480-482. URL: http://doi. wiley.com/10.1002/qj.49710042520. doi:10.1002/qj.49710042520.

Hoskins, B. J., McIntyre, M. E., \& Robertson, A. W. (1985). On the use and significance of isentropic potential vorticity maps. Quarterly Journal of the Royal Meteorological Society, 111, 877-946. doi:10.1002/qj . 49711147002.

Hua, B. L., Ménesguen, C., Le Gentil, S., Schopp, R., Marsset, B., \& Aiki, H. (2013). Layering and turbulence surrounding an anticyclonic oceanic vortex: in situ observations and quasi-geostrophic numerical simulations. Journal of Fluid Mechanics, 731, 418-442. doi:10.1017/jfm.2013.369.

Isern-Fontanet, J., Lapeyre, G., Klein, P., Chapron, B., \& Hecht, M. W. (2008). Threedimensional reconstruction of oceanic mesoscale currents from surface information. Journal of Geophysical Research: Oceans, 113, C9. doi:10.1029/2007JC004692.

Keppler, L., Cravatte, S., Chaigneau, A., Pegliasco, C., Gourdeau, L., \& Singh, A. (2018). Observed Characteristics and Vertical Structure of Mesoscale Eddies in the Southwest 
Tropical Pacific. Journal of Geophysical Research: Oceans, 123, 2731-2756. doi:10.1002/ 2017 JC013712.

Klein, P., Hua, B. L., Lapeyre, G., Capet, X., Le Gentil, S., \& Sasaki, H. (2008). Upper Ocean Turbulence from High-Resolution 3D Simulations. Journal of Physical Oceanography, 38, 1748-1763. doi:10.1175/2007JP03773.1.

Large, W. G., McWilliams, J. C., \& Doney, S. C. (1994). Oceanic vertical mixing: A review and a model with a nonlocal boundary layer parameterization. Reviews of Geophysics, 32, 363-403. doi:10.1029/94RG01872.

Le Vu, B., Stegner, A., \& Arsouze, T. (2018). Angular Momentum Eddy Detection and Tracking Algorithm (AMEDA) and Its Application to Coastal Eddy Formation. Journal of Atmospheric and Oceanic Technology, 35, 739-762. doi:10.1175/JTECH-D-17-0010.1.

Lévy, M., Franks, P. J. S., \& Smith, K. S. (2018). The role of submesoscale currents in structuring marine ecosystems. Nature Communications, 9, 4758. doi:10.1038/ s41467-018-07059-3.

L'Hégaret, P., Beal, L. M., Elipot, S., \& Laurindo, L. (2018). Shallow Cross-Equatorial Gyres of the Indian Ocean Driven by Seasonally Reversing Monsoon Winds. Journal of Geophysical Research: Oceans, (pp. 8902-8920). doi:10.1029/2018JC014553.

L'Hégaret, P., Carton, X., Louazel, S., \& Boutin, G. (2016). Mesoscale eddies and submesoscale structures of Persian Gulf Water off the Omani coast in spring 2011. Ocean Science, 12, 687-701. doi:10.5194/os-12-687-2016.

L'Hégaret, P., Duarte, R., Carton, X., Vic, C., Ciani, D., Baraille, R., \& Corréard, S. (2015). Mesoscale variability in the Arabian Sea from HYCOM model results and observations: impact on the Persian Gulf Water path. Ocean Science, 11, 667-693. doi:10.5194/os-11-667-2015.

Li, L., Nowlin, W. D., \& Jilan, S. (1998). Anticyclonic rings from the Kuroshio in the South China Sea. Deep Sea Research Part I: Oceanographic Research Papers, 45, 1469-1482. doi:10.1016/S0967-0637(98)00026-0. 
McDougall, T. J., \& Barker, P. M. (2011). Getting started with TEOS-10 and the Gibbs Seawater (GSW), Oceanographic Toolbox.

McWilliams, J. C. (1985). Submesoscale, coherent vortices in the ocean. Reviews of Geophysics, 23, 165-182. doi:10.1029/RG023i002p00165.

McWilliams, J. C., Gent, P. R., \& Norton, N. J. (1986). The Evolution of Balanced, LowMode Vortices on the $\beta$-Plane. Journal of Physical Oceanography, 16, 838-855. doi:https: //doi .org/10.1175/1520-0485(1986)016<0838: TEOBLM>2 . 0. CO;2.

Ménesguen, C., Le Gentil, S., Marchesiello, P., \& Ducousso, N. (2018). Destabilization of an Oceanic Meddy-Like Vortex: Energy Transfers and Significance of Numerical Settings. Journal of Physical Oceanography, 48, 1151-1168. doi:10.1175/jpo-d-17-0126.1.

Meunier, T., Ménesguen, C., Carton, X., Le Gentil, S., \& Schopp, R. (2018). Optimal perturbations of an oceanic vortex lens. Fluids, 3, 63. doi:10.3390/fluids3030063.

Meunier, T., Ménesguen, C., Schopp, R., \& Le Gentil, S. (2015). Tracer Stirring around a Meddy: The Formation of Layering. Journal of Physical Oceanography, 45, 407-423. doi:10.1175/JPO-D-14-0061.1.

Meunier, T., Pallas-Sanz, E., Ruiz-Angulo, A., Ochoa-de-la-Torre, J., \& Tenreiro, M. (2019). Observations of Layering under an Anticyclonic Warm-Core Ring in the Gulf of Mexico. Journal of Physical Oceanography, doi:10.1175/JPO-D-18-0138.1.

Meunier, T., Pallas-Sanz, E., Tenreiro, M., Portela, E., Ochoa, J., Ruiz-Angulo, A., \& Cusí, S. (2018). The Vertical Structure of a Loop Current Eddy. Journal of Geophysical Research: Oceans, 123,6070-6090. doi:10.1029/2018JC013801.

Morel, Y., \& McWilliams, J. (2001). Effects of Isopycnal and Diapycnal Mixing on the Stability of Oceanic Currents. Journal of Physical Oceanography, 31, 2280-2296. doi:10. $1175 / 1520-0485(2001) 031<2280:$ EOIADM $>2.0$. CO 2.

Morel, Y. G., Darr, D. S., \& Talandier, C. (2006). Possible Sources Driving the Potential Vorticity Structure and Long-Wave Instability of Coastal Upwelling and Downwelling Currents. Journal of Physical Oceanography, 36, 875-896. doi:10.1175/JP02899.1. 
Nadiga, B. T. (2014). Nonlinear evolution of a baroclinic wave and imbalanced dissipation. Journal of Fluid Mechanics, 756, 965-1006. doi:10.1017/jfm.2014.464.

Nguyen, H. Y., Hua, B. L., Schopp, R., \& Carton, X. (2012). Slow quasigeostrophic unstable modes of a lens vortex in a continuously stratified flow. Geophysical $\mathscr{G}$ Astrophysical Fluid Dynamics, 106, 305-319. doi:10.1080/03091929.2011.620568.

Olson, D. B., \& Evans, R. H. (1986). Rings of the Agulhas current. Deep Sea Research Part A. Oceanographic Research Papers, 33, 27-42. doi:10.1016/0198-0149(86)90106-8.

Pedlosky, J. (1964). The Stability of Currents in the Atmosphere and the Ocean: Part I. Journal of the Atmospheric Sciences, 21, 201-219. doi:10.1175/1520-0469(1964) 021<0201: TSOCIT>2.0.CO;2.

Pedlosky, J. (2013). Geophysical Fluid Dynamics. Springer Science \& Business Media.

Penven, P., Halo, I., Pous, S., \& Marié, L. (2014). Cyclogeostrophic balance in the Mozambique Channel. Journal of Geophysical Research: Oceans, 119, 1054-1067. doi:10.1002/2013JC009528.

Richardson, P. L. (1983). Gulf Stream Rings, In A. R. Robinson (Ed.), Eddies in Marine Science (pp. 19-45). Berlin, Heidelberg: Springer Berlin Heidelberg. doi:10.1007/ 978-3-642-69003-7_2.

Riedinger, X., \& Gilbert, A. D. (2014), Critical layer and radiative instabilities in shallowwater shear flows. Journal of Fluid Mechanics, 751, 539-569. doi:10.1017/jfm. 2014.303.

Ripa, P. (1991). General stability conditions for a multi-layer model. Journal of Fluid Mechanics, 222, 119-137. doi:10.1017/S0022112091001027.

Roullet, G., Capet, X., \& Maze, G. (2014). Global interior eddy available potential energy diagnosed from Argo floats. Geophysical Research Letters, 41, 1651-1656. doi:10.1002/ 2013GL059004.

Scharffenberg, M. G., \& Stammer, D. (2010). Seasonal variations of the large-scale geostrophic flow field and eddy kinetic energy inferred from the TOPEX/Poseidon and 
Jason-1 tandem mission data. Journal of Geophysical Research, 115, C2. doi:10.1029/ 2008JC005242.

Schott, F. A., \& McCreary, J. P. (2001). The monsoon circulation of the Indian Ocean. Progress in Oceanography, 51, 1-123. doi:10.1016/S0079-6611(01)00083-0.

Shchepetkin, A. F., \& McWilliams, J. C. (2005). The regional oceanic modeling system (ROMS): a split-explicit, free-surface, topography-following-coordinate oceanic model. Ocean Modelling, 9, 347-404. doi:10.1016/j.ocemod.2004.08.002.

Shchepetkin, A. F., \& McWilliams, J. C. (2011). Accurate Boussinesq oceanic modeling with a practical, "Stiffened" Equation of State. Ocean Modelling, 38, 41-70. doi:10.1016/j . ocemod.2011.01.010.

Snyder, C., Skamarock, W. C., \& Rotunno, R. (1993). Frontal dynamics near and following frontal collapse. Journal of the atmospheric sciences, 50, 3194-3212. doi:10.1175/ $1520-0469$ (1993) 050<3194: FDNAFF $>2.0 . \mathrm{CO} ; 2$.

Stegner, A., \& Dritschel, D. G. (2000). A Numerical Investigation of the Stability of Isolated Shallow Water Vortices. Journal of Physical Oceanography, 30, 2562-2573. doi:10.1175/ $1520-0485(2000) 030<2562$ : ANIOTS $>2$. 0 . CO $; 2$.

Thomas, L. N., Taylor, J. R., Ferrari, R., \& Joyce, T. M. (2013). Symmetric instability in the Gulf Stream. Deep Sea Research Part II: Topical Studies in Oceanography, 91, 96-110. doi:10.1016/j.dsr2.2013.02.025.

Vallis, G. K. (2017). Atmospheric and oceanic fluid dynamics. Cambridge University Press.

Vic, C., Roullet, G., Carton, X., \& Capet, X. (2014). Mesoscale dynamics in the Arabian Sea and a focus on the Great Whirl life cycle: A numerical investigation using ROMS. Journal of Geophysical Research: Oceans, 119, 6422-6443. doi:10.1002/2014JC009857.

Yim, E., \& Billant, P. (2015). On the mechanism of the Gent-McWilliams instability of a columnar vortex in stratified rotating fluids. Journal of Fluid Mechanics, 780, 5-44. doi:10.1017/jfm.2015.426. 
892

Young, W. R., \& Chen, L. (1995). Baroclinic Instability and Thermohaline Gradient Alignment in the Mixed Layer. Journal of Physical Oceanography, 25, 3172-3185. doi:10.1175/1520-0485(1995)025<3172:BIATGA>2 . 0 . CO;2.

Zhang, Z., Wang, W., \& Qiu, B. (2014). Oceanic mass transport by mesoscale eddies. Science, 345, 322-324. doi:10.1126/science.1252418. 
Charly de Marez: Conceptualization, Methodology, Software, Writing- Original draft. Thomas Meunier: Conceptualization, Methodology, Writing- Reviewing and Editing. Mathieu Morvan: Software, Writing- Reviewing and Editing.

Pierre L'Hégaret: Writing- Reviewing and Editing.

Xavier Carton: Writing- Reviewing and Editing, Supervision, Funding acquisition. 
All co-authors have seen and agree with the contents of the manuscript and there is no financial interest to report. We certify that the submission is original work and is not under review at any other publication.

Charly de Marez and co-authors 